\title{
Integration durch Partizipation Soziale Bewegungen im politischen System der USA
}

Der Beitrag analysiert Interaktionsprozesse und Entwicklungsdynamiken im System der intermediären Interessenvermittlung der USA. Mit Blick auf die Neuen Sozialen Bewegungen und die Protestbewegung der Christlichen Rechten werden folgende Thesen erhärtet: Soziale Bewegungen haben einen Anreiz, Interessengruppen auszubilden, nicht jedoch, sich als Partei zu formieren. Sie haben einen Anreiz, Parteien zu infiltrieren, um auf deren Kandidatenrekrutierung und Programmformulierung Einfluss zu nehmen. Für Parteien ihrerseits besteht ein Anreiz, soziale Bewegungen zu kooptieren, um sich deren Anhänger als Wähler zu versichern. Soziale Bewegungen bewirken - zumindest phasenweise - eine wachsende ideologische Polarisierung im amerikanischen Parteiensystem, die zu einer weiterreichenden Re-Konfiguration der bestehenden Wählerkoalitionen führt. Langfristig jedoch wirken die zentripetalen Kräfte des Systems mäßigend. 


\section{Inhalt}

1. Die Vermittlung von Interessen- und Wertkonflikten im politischen System der USA

2. Die Hauptelemente des intermediären Systems

3. Interaktionsprozesse und Entwicklungsdynamiken im System der intermediären Interessenvermittlung der USA - Vier Thesen

a) Interessengruppenbildung

b) Verzicht auf Parteigründung

c) Infiltration von Parteien

d) Kooptation durch Parteien

4. Die Thesen auf dem Prüfstand

a) Neue soziale Bewegungen

b) Die Christliche Rechte

5. Die Folgen für das Parteiensystem 260

6. Schluss

\section{Die Vermittlung von Interessen- und Wertkonflikten im politischen System der USA}

Das politische System der USA ist als präsidentielle Demokratie und föderative Republik zu charakterisieren (Lowi 2006; Jäger/Haas/Welz 2007). Ein hohes Maß an Dezentralisierung, Machtaufteilung und Gewaltenbegrenzung, ja Zersplitterung von Einfluss und Zuständigkeiten gehört zu seinen spezifischen Merkmalen (Lösche 1989: 63). Zugleich ist es überaus partizipationsorientiert, d. h. auf die politische Beteiligung von sozialen Gruppen und Assoziationen hin angelegt. Beides, die institutionelle Fragmentierung wie auch die strukturelle Offenheit für die Einflussnahme von sozialen Gruppen, war von den Verfassungsvätern gewollt. Nach ihrer Überzeugung konnte nur eine Vielzahl politisch aktiver Gruppen und „Parteiungen“ (factions) die Freiheitlichkeit der großräumigen Republik dauerhaft sichern. Die Gründung solcher Gruppen sollte deshalb nicht durch institutionelle Hürden erschwert werden. Der ihnen tendenziell innewohnenden Gemeinwohlfeindlichkeit glaubte man weitaus wirksamer durch die großzügige Möglichkeit zu ihrer Proliferation begegnen zu können. Der daraus sich ergebende intensive Wettbewerb um politischen Einfluss und politische Macht müsste, so die Überlegung, die Durchsetzungschancen jeder einzelnen Gruppe deutlich reduzieren. Zudem 
würde die vertikale wie horizontale Teilung der staatlichen Gewalten verhindern, dass sich eine faction - sollte sie doch einmal eine Mehrheit hinter sich bringen können - auf allen Ebenen des politischen Systems durchsetzen könnte:

„The influence of factious leaders may kindle a flame within their particular States but will be unable to spread a general conflagration through the other States. A religious sect may degenerate into a political faction in a part of the Confederacy; but the variety of sects dispersed over the entire face of it must secure the national councils against any danger from that source. A rage for $[\ldots]$ an abolition of debts, for an equal division of property $[\ldots]$ will be less apt to pervade the whole body of the Union than a particular member of it, in the same proportion as such a malady is more likely to taint a particular county or district than an entire State. In the extent and proper structure of the Union, therefore, we behold a republican remedy for the diseases most incident to republican government" (Hamilton/ Madison/Jay, Federalist Papers, Nr. 10).

Diese Argumentation Madisons im 10. Federalist hat die ideologische Akzeptanz des Interessengruppenpluralismus in den USA historisch begründet. Die Bildung von Vereinigungen zur Durchsetzung partikularer Ziele und die Inanspruchnahme der konstitutionell verankerten Rede- und Meinungsfreiheit zur Artikulation gruppenspezifischer Interessen wurden seit der Gründung der Republik als legitime Formen der gesellschaftlichen Einflussnahme auf staatliche Entscheidungen angesehen (Wasser 2007: 327 f.).

Das, was der 10. Federalist noch pejorativ ,parties“ oder „factions“ nannte, ist im Folgenden als „soziale Bewegungen“, „Interessengruppen“ und „Parteien“ näher zu bestimmen. Sie bilden heute die drei Hauptelemente des intermediären Systems der Interessenvermittlung ${ }^{1}$ im politischen System der USA.

1 Zum Begriff vgl. Rucht 1993: 257 f.: „,Das spezifische Merkmal intermediärer Systeme politischer Interessenvermittlung besteht darin, das politisch-administrative System, verstanden als Produktionsort gesamtgesellschaftlich verbindlicher Entscheidungen, mit den in ihre Sozialmilieus und Kleingruppen eingebetteten Individuen zu vermitteln." 


\section{Die Hauptelemente des intermediären Systems}

Unter „sozialen Bewegungen“ wird hier mit Rucht (1994: 22 f., 76 f.) ein auf gewisse Dauer gestellter Versuch mobilisierter Netzwerke von Gruppen und Organisationen verstanden, sozialen Wandel durch Protest - notfalls bis hin zur Gewaltanwendung - herbeizuführen, zu verhindern oder rückgängig zu machen. Soziale Bewegungen verfügen sowohl über aktive, zur Mobilisierung und Lenkung befähigte Führungspersönlichkeiten oder Führungsgruppen („Bewegungsunternehmer“) als auch über einen größeren - in unterschiedlichen Graden - leicht mobilisierbaren Anhang aus Unterstützern und Sympathisanten. Sie weisen eine organisatorische Basis auf, sind jedoch in ihrer Gesamtheit keine Organisation, da ihnen verbindliche Kriterien der Mitgliedschaft, eine interne Kompetenzaufteilung und gemeinsame Regelungen zur Entscheidungsfindung fehlen (Rucht 1993: 263 f.).

Die Reichweite ihrer Veränderungsabsichten kann sehr groß (Einführung einer ökologischen Wirtschaftsordnung) oder sehr begrenzt (Rücknahme eines Gesetzes) sein. Bevorzugte Aktionsformen sind Protesthandlungen wie Demonstrationen, Boykotte, Sit-ins, Unterschriftensammlungen und öffentliche Appelle, die McAdam (1999: 25) zusammenfassend als ,noninstitutional forms of political participation“ bezeichnet. Definitionsgemäß spricht man dann nicht mehr von dem Vorhandensein sozialer Bewegungen, „wenn diese entweder dazu übergegangen sind, systematischen Lobbyismus oder pressure group politics zu betreiben ( $\rightarrow$ Interessengruppen), wenn sie in unverbundene Kleingruppenaktivitäten zerfallen oder wenn sie zu Einzelaktionen einer herausragenden Person zusammengeschrumpft sind" (Nedelmann 1995: 330).

„Interessengruppen“ bezeichnen dagegen einen auf formaler Mitgliedschaft und ausdifferenzierter Binnenstruktur beruhenden Verband, der die Interessen einer bestimmten Gesellschaftsgruppe artikuliert, bündelt und gegenüber den Mitgliedern, anderen Organisationen, der Öffentlichkeit und vor allem gegenüber der Regierung, der Verwaltung und den Gerichten - des Bundes wie der Einzelstaaten - durch direkte oder indirekte Einflussnahme oder Druckausübung (pressure groups) vertritt (Schmidt 1995: 435 f.). Interessengruppen weisen gegenüber sozialen Bewegungen eine festere organisatorische Verfassung, einen höheren Grad an Rollenspezifikation sowie eine größere Kontinuität und Stabilität auf. Meist regelt eine förmliche Satzung, ein mission statement o. Ä. Zielsetzung, Mitgliedschaft, Finanzierung und den modus operandi. Interessengruppen zielen wie soziale Bewegungen und 
Parteien auf politische Einflussnahme, unterscheiden sich von Ersteren jedoch durch den stärker formalisierten Charakter ihrer Aktivitäten, von Letzteren durch die größere Partikularität ihrer Interessen und die Begrenztheit ihrer Agenden. Zudem richtet sich ihr Bestreben nicht auf die Übernahme politischer Verantwortung in Parlament und Regierung, weshalb sie in Wahlen keine Kandidaten für Ämter und Mandate aufstellen. Ihre bevorzugten Aktionsformen sind das Lobbying, die Unterstützung ,befreundeter“ Kandidaten oder Parteien in Wahlkämpfen sowie die Durchführung von Themenkampagnen in den Medien (issue campaigns) und andere Spielarten der Öffentlichkeitsarbeit.

Interessengruppen sind in den USA von staatlicher Seite anerkannt: Ihre Beteiligung am parlamentarischen Willensbildungs- und Entscheidungsprozess ist durch Hearings vor den Ausschüssen und Unterausschüssen des Kongresses institutionalisiert; vor den Bundesgerichten ist ihre Mitwirkung durch die Möglichkeit schriftlicher Stellungnahmen (amicus curiae briefs) garantiert. Ihre gesetzliche (und steuerrechtliche) Behandlung variiert, je nachdem, ob sie als Lobbyisten, als political action committees (PACs), als „Ideenagenturen“ (think tanks) oder „politische Bildungseinrichtungen“ operieren (Berry 1997): Lobby-Aktivitäten von Interessengruppen im Umkreis des Kongresses sind legal, unterliegen aber einer gesetzlich geregelten Registrierungs- und Offenlegungspflicht. Political action committees dürfen direkte Wahlkampfspenden an Kandidaten leisten, müssen aber gesetzlich festgelegte Höchstgrenzen beachten. Interessengruppen, die die Öffentlichkeit „,bilden“ und „,informieren“ wollen, gelten als gemeinnützig und sind - im Unterschied etwa zu den PACs - von der Steuer befreit; Spenden an sie sind steuerabzugsfähig. Dafür sind ihnen jedoch Wahlkampf- oder ausgedehntere Lobby-Aktivitäten untersagt.

Soziale Bewegungen entgehen weitgehend den gesetzlichen Beschränkungen, denen die Aktivitäten von Interessengruppen unterliegen, doch können sie im Gegenzug auch viele der Verbänden gewährten Steuerprivilegien und institutionalisierten Mitwirkungsmöglichkeiten kaum nutzen. Aufgrund ihrer fluiden Gestalt und ihrer meist nur rudimentär entwickelten organisatorischen Struktur sind sie für staatliche Institutionen zu wenig greifbar, um als Informationsquelle oder Adressaten offizieller Begünstigungen und Förderungen infrage zu kommen.

„Parteien“ schließlich sind - wie Interessengruppen - organisierte Zusammenschlüsse gleich gesinnter Bürger zur Förderung und Durchsetzung gemeinsamer politischer Anliegen, die sie jedoch - anders als jene - vor 
allem durch Ämtererwerb und direkte politische Gestaltung anstreben. Parteien übernehmen in modernen politischen Systemen zahlreiche Funktionen wie die der politischen Zielfindung, der Artikulation und Aggregation divergierender gesellschaftlicher Interessen, der Mobilisierung und politischen Sozialisierung der Bürger, der Elitenrekrutierung und Regierungsbildung, der Konfliktregulierung und Konsensfindung, der Legitimitätsbeschaffung und der Machtkontrolle.

Während diese Funktionen in parlamentarischen Systemen weitgehend gleichrangig erfüllt werden, dominieren im präsidentiellen System der USA die Elitenrekrutierungs- und Wählermobilisierungsfunktion der Parteien (Klumpjan 1998): Da sie für die Regierungsbildung im engeren Sinne hier nicht erforderlich sind und andere Institutionen und Organisationen (wie Interessengruppen und Medien) zahlreiche der genannten Aufgaben übernehmen, treten diese bei den amerikanischen Parteien hinter die elektoralen Funktionen zurück (,,a party is to elect"). Selbst in diesem beschränkten Bereich mussten sie allerdings im Laufe der letzten Jahrzehnte einige Funktionsverluste hinnehmen: So ist die Wahlkampffinanzierung immer stärker in die Hände der Kandidaten selbst bzw. der sie unterstützenden political action committees übergegangen, haben Interessengruppen die Aufgabe der Information und Mobilisierung der Wahlberechtigten (gelegentlich auch die Kandidatenrekrutierung) partiell mit übernommen, prägen medieninduzierte Aufmerksamkeitszyklen für bestimmte issues und die individuellen Stellungnahmen der Kandidaten selbst die Wahrnehmung ihres politischen Profils weit stärker als die Prioritätensetzung und Zielformulierung ihrer Parteien (Lösche 2007; Bibby 2003).

Doch nicht nur in funktionaler, auch in struktureller und programmatischer Hinsicht unterscheiden sich amerikanische Parteien von europäischen: Amerikanische Parteien sind lockere Koalitionen aus sehr unterschiedlichen sozialen, ethnischen und religiösen Bevölkerungsgruppen, lose Föderationen aus lokalen, einzelstaatlichen und nationalen Komitees (wobei hier in den letzten Jahren durch die Stärkung der Bundesparteien allerdings ein Trend zur Zentralisierung und Nationalisierung zu beobachten ist, der von einer zunehmenden Verflechtung der verschiedenen Parteiebenen begleitet wird; Lösche 2007). Sie kennen weder eine formelle Parteimitgliedschaft noch sind sie hierarchisch aufgebaut. Voll ausgebildete Parteistrukturen werden meist nur während der Präsidentschaftswahlen auf allen Ebenen des politischen Systems erkennbar. Auch programmatisch sind sie relativ offen. Die 
Parteiprogramme dienen weniger der Festlegung der eigenen Amts- und Mandatsträger auf bestimmte policies als vielmehr der Profilierung und Selbstdarstellung sowie der Koalitionsbildung ,nach innen“: Die Programme sollen die disparate „Parteibasis“ zusammenhalten, indem sie „verbindende“ Werte und Zielvorstellungen artikulieren. Entsprechend vage formuliert und breit gefächert fallen sie aus.

Interessengruppen wenden sich in den USA - anders als in parlamentarischen Systemen - kaum an die „schwachen“ Parteien, um ihren Anliegen Gehör und Geltung zu verschaffen. Ihre Ansprechpartner sind die Kandidaten und Abgeordneten selbst, insbesondere die Vorsitzenden der für sie wichtigen parlamentarischen Ausschüsse und Unterausschüsse - meist ohne Rücksicht auf deren jeweilige Parteizugehörigkeit. Da sich diese ihren Wählern, Spendern und Förderern mehr verpflichtet fühlen als ihrer Partei, ist der Versuch, sie durch persönliche Kontaktpflege, die Mobilisierung ihrer Wahlkreise (grassroots lobbying) oder die finanzielle Unterstützung ihrer Wahlkampagnen zu beeinflussen, Erfolg versprechender als die Einflussnahme auf ihre Partei. Interessengruppen durchdringen deshalb auch nicht, wie etwa im deutschen System, Parlament und Fraktionen, sondern wirken ,von außen“ auf die Kongressmitglieder ein (Lösche 2004; Wasser 2007).

\section{Interaktionsprozesse und Entwicklungsdynamiken im System der intermediären Interessenvermittlung der USA - Vier Thesen}

Die folgenden Thesen können für die Entwicklung von sozialen Bewegungen $^{2}$ im amerikanischen System der Interessenvermittlung ${ }^{3}$ aufgestellt werden, soweit sie einer instrumentellen Handlungslogik gehorchen und eine

2 Zur Bewegungsforschung insgesamt vgl. Rucht 1991, 1994; Morris/Mueller 1992; McAdam/McCarthy/Zald 1996; Meyer/Tarrow 1998; Costain/McFarland 1998; Klein/Legrand/Leif 1999; Freeman/Johnson 1999; Goldstone 2003; Snow/Soule/ Kriesi 2004; Meyer/Jenness/Ingram 2005; Kolb 2007.

3 Das horizontal wie vertikal stark fragmentierte Entscheidungssystem der USA ist relativ offen für die Einflussnahme von sozialen Bewegungen, was deren Entstehung begünstigt. Die Fähigkeit und Bereitschaft des Staates zu repressiven Maßnahmen gegenüber neuen Bewegungen ist zudem gering, selbst wenn sie mit unkonventionellen Mitteln operieren. Darüber hinaus ist die Geschlossenheit der Status-quo-Eliten zu wenig ausgeprägt, als dass neue Bewegungen keine Bündnispartner finden könnten (McAdam 1996: 27). 
allgemeine Strategie der Machtorientierung verfolgen (d. h. auf politische Beteiligung, Verhandlung, Druck und Konfrontation zielen). ${ }^{4}$

\section{a) Interessengruppenbildung}

These 1: Soziale Bewegungen haben einen Anreiz, Interessengruppen auszubilden.

Nach Rucht (1994: 314) ergeben sich starke verbandsförmige Mobilisierungsstrukturen innerhalb von Bewegungen am ehesten a) bei einem Entscheidungssystem, das strukturell für lobbyistische und klientelistische Beziehungen offen und auf Verflechtungen mit Verbänden angelegt ist, b) wenn die Bewegungsthemen durch bereits etablierte Verbände nicht oder nur schwach besetzt sind, und c) bei einer konfliktfreudigen politischen Kultur in Verbindung mit einem partizipatorischen Politikstil. „Insgesamt“, so Rucht (1994: 316), ist „die Kontextstruktur in den USA einer verbandsförmigen Interessenvertretung innerhalb und außerhalb von Bewegungen förderlich.“

$\mathrm{Zu}$ den pull-Faktoren, die sozialen Bewegungen die Wahl einer verbandlichen Organisationsform bzw. die Ausgründung von Interessengruppen nahe legen, sind allgemein jene institutionellen und rechtlichen Rahmenbedingungen des politischen Systems bzw. jene gesetzlichen Bestimmungen zu rechnen, die in der Literatur unter der Bezeichnung ,institutional channelling of social movements" (McCarthy/Britt/Wolfson 1991) diskutiert werden. Diese staatlichen Mechanismen der „Kanalisierung“ verschaffen solchen Gruppierungen Vorteile, die bestimmte staatlich anerkannte Organisations- und Aktionsformen wählen. ${ }^{5}$ Dazu gehören etwa steuerrechtliche Vergünstigungen für jene registrierten Organisationen, die das Ziel der politischen Bildung und Informationsvermittlung verfolgen: Eine spezifische Organisationsstruktur, die eine zweckgemäße Verwendung der eingeworbenen Mittel erwarten lässt, bildet hier die Voraussetzung für die Gewährung der Steuerprivilegien. Zwar ist damit zugleich die Auflage verbunden, auf bestimmte politische (insbesondere parteipolitische) Aktivitäten zu verzichten, die sozialen Bewegungen sonst offen stünden; doch können Organisationen, die als gemeinnützig anerkannt und von der Einkommensteuer befreit sind, derartige

4 Sie gelten nicht für Bewegungen, die einer expressiven Handlungslogik folgen, der eine allgemeine Strategie der Identitätsbehauptung (durch demonstrative Verhaltensabweichung, subkulturellen Rückzug oder gegenkulturelle Herausforderung) entspricht (wie z. B. Teile der Homosexuellenbewegung). Zur Systematik der Handlungslogiken (,,instrumentell“"vs. „expressiv“) einer Bewegung vgl. Rucht 1994: 89.

Gleichzeitig reduzieren sie die Kontrollkosten des Staates. 
Aktivitäten durchführen, wenn sie entsprechende rechtlich eigenständige Unterorganisationen gründen.

Ferner ist die Aussicht, als registrierte Interessengruppe regelmäßig Einladungen zu Anhörungen in den Ausschüssen der Parlamente auf Bundesund Einzelstaatenebene oder einen verbesserten Zugang zu den politischen Entscheidungsträgern zu erhalten, auf diese Weise frühzeitig Informationen über Gesetzesvorhaben gewinnen und eigene Standpunkte in die Beratungen einbringen zu können, ein weiterer Anreiz zur Ausbildung stabiler, deutlich strukturierter und formalisiert agierender Interessengruppen. Nur kontinuierlich arbeitende Verbände mit einer klaren Rollenspezifikation sind für Abgeordnete interessant, denn allein mit ihnen können verlässliche und vorteilhafte „do-ut-des“-Beziehungen des Informationsaustauschs aufgebaut werden. Kontinuierliche und professionelle Arbeit ist zudem die Voraussetzung für den Zugang zu den relevanten issue networks und policy communities, in denen Experten über zukünftige Politiken beraten und Entscheidungen der Parlamente und Bürokratien vorstrukturieren. Deren Einfluss beruht nicht zuletzt darauf, dass aus ihrem Kreis regelmäßig hohe Regierungsbeamte oder politische Berater rekrutiert werden. ${ }^{6}$

Wenn eine Organisation langfristig angelegt ist, steigt darüber hinaus vor den Bundesgerichten und insbesondere dem U. S. Supreme Court die Wahrscheinlichkeit, zu Verfahren als amicus curiae zugelassen oder von einer Prozesspartei um Unterstützung gebeten zu werden (und somit Einfluss auf die Rechtsprechung und Rechtsentwicklung in einem Policy-Bereich nehmen zu können). Nur dadurch kann sie sich einen Namen als glaubwürdige, juristisch qualifizierte Repräsentantin bestimmter Interessen oder Positionen machen. Die Unterstützung von Klagen vor Bundesgerichten durch bekannte, nationale Interessengruppen erhöht, wie Untersuchungen zeigen (z. B. Caldeira/Wright 1998), die Erfolgsaussichten. Interessengruppen können zudem selbst Musterprozesse anstrengen, finanzieren oder betreuen und dadurch politischen Einfluss nehmen. Der Weg des court lobbying durch juridische Organisationen bietet sich insbesondere dort an, wo soziale Bewegungen in der Öffentlichkeit auf starke Ablehnung stoßen und bewegungsförmige Protestaktivitäten wie Demonstrationen und Massenkundgebungen eher dazu führen, die Gegner ihrer Reformziele zu mobilisieren. Die Aktivitäten von

6 Heclo 1978. Ob Vertreter bewegungsnaher Interessengruppen für politische Ämter rekrutiert werden, hängt allerdings von weiteren Kontextfaktoren ab, etwa der grundsätzlichen politischen Position einer Regierung im jeweiligen Politikfeld (Imig 1998). 
juridischen Interessengruppen erlauben es dagegen, von der Öffentlichkeit weitgehend unbeachtet die eigenen politischen Ziele zu verfolgen.

Letzteres leitet über zur Diskussion der Bedeutung von push-Faktoren, die den Bewegungseliten die Wahl einer verbandlichen Organisationsform nahe legen: Eine erfolgreiche Strategieanwendung erfordert ein hohes Maß an Informationsbeschaffung und interner Handlungskoordination: Der Aufbau fester Organisationsstrukturen und einer registrierten Mitgliedschaft, die gezielt mobilisiert werden kann, die Erschließung langfristig ergiebiger Finanzierungsquellen und die Errichtung geeigneter Informationsverarbeitungskapazitäten etwa in Form fest angestellter, fachlich qualifizierter Mitarbeiter steigern die Handlungs- und Strategiefähigkeit von Bewegungen, weshalb diese zur Bildung von Interessengruppen (bzw. zur Fortentwicklung ihrer Bewegungsorganisationen zu Interessengruppen) stimuliert werden.

Auch verfügen in der Regel nur Interessengruppen über die Fähigkeit, professionelle Öffentlichkeitsarbeit zu betreiben und dadurch die Aufmerksamkeit der Medien langfristig auf bestimmte Positionen und Forderungen $\mathrm{zu}$ lenken. Institutionalisierte Interessengruppen mit einem klaren programmatischen Profil werden von den Medien weit eher wahrgenommen und als Informationsquellen kontaktiert (und ihre Repräsentanten weit eher zu den beliebten agonalen politischen Talkshows der Fernsehsender eingeladen) als Vertreter stark fluider sozialer Bewegungen oder Bewegungsorganisationen mit diffuser oder weit ausgreifender Agenda.

These 1.1: Sind Bewegungsthemen durch bereits etablierte Verbände besetzt, werden diese von einer Bewegung dann infiltriert und übernommen, wenn die Bewegung insgesamt ressourcenstärker ist und über eine größere Zahl aktiver Mitglieder verfügt.

Sind die in These 1 ausgeführten Kriterien a), b) und c) erfüllt, führt dies bei sozialen Bewegungen zur Ausbildung eigener Interessenorganisationen. Trifft Kriterium b) nicht zu, existieren also bereits Verbände, die Bewegungsthemen aktiv vertreten, wird die Bewegung diese zu übernehmen versuchen: Denn soziale Bewegungen können ihre Organisationskosten senken, indem sie sich bereits bestehende Verbände einverleiben. Dadurch erhalten sie die Verfügungsgewalt über deren Ressourcen, insbesondere auch über deren (,Marken“-)Namen, unter dem sie fortan operieren und etwa Spendengelder einwerben können.

These 1.2: Der gemäßigt partizipationsorientierte Politikstil der USA fördert auf lange Sicht die Professionalisierung der Arbeit 
politischer Organisationen und verbessert dadurch ihre Durchschlagskraft.

Bilden soziale Bewegungen Interessengruppen aus, so wird der gemäßigt partizipationsorientierte Politikstil der USA auf lange Sicht zu einer Verbesserung der Organisationsarbeit beitragen (Rucht 1994: 315). Die regelmäßige Nutzung politischer Einflusskanäle in Form von Verfahrensbeteiligung und Lobbying wird bei den Akteuren durch Lerneffekte $\mathrm{zu}$ einer Steigerung ihrer Fähigkeiten sowie einer effektiveren Verwendung vorhandener Ressourcen führen. Die dadurch freigesetzten Mittel können für zusätzliche politische Maßnahmen verwendet werden, womit die Durchschlagskraft der Organisationen steigt. Die Chancen, öffentliche Aufmerksamkeit zu wecken und Zugang zu politischen Entscheidungsträgern und politischen Verfahren zu gewinnen, verbessern sich, was ceteris paribus die Wahrscheinlichkeit einer Durchsetzung der eigenen Forderungen und Positionen im politischen Prozess erhöht.

\section{b) Verzicht auf Parteigründung}

These 2: Soziale Bewegungen haben in den USA keinen Anreiz, sich als Partei zu formieren.

Amerikanische Parteien sind in funktionaler, struktureller und programmatischer Hinsicht im Vergleich zu europäischen, wie dargestellt, recht schwache Gebilde. ${ }^{7}$ Das liegt zum einen an der Institutionen-Ordnung und föderativen Struktur des amerikanischen politischen Systems, zum anderen an historischen Entwicklungen wie der Einschränkung der Patronagemöglichkeiten der Parteien durch politische und administrative Reformen, die fast flächendeckende Einführung von Vorwahlen, die Reform des Wahlkampffinanzierungsgesetzes und die zunehmende Bedeutung des Fernsehens als Informationsvermittler und politische Sozialisationsagentur. Infolgedessen kam es in den letzten Jahrzehnten selbst in jenen Bereichen, in denen amerikanische Parteien genuine Aufgaben zu erfüllen haben, zu Funktionsverlusten: "Candidate centered (nominating) campaigns" sind an die Stelle der traditionellen ,party centered campaigns“" getreten (Wattenberg 1991).

Darüber hinaus können Parteien in den Vereinigten Staaten aufgrund des relativen Mehrheitswahlsystems nur als breite Koalitionen unterschiedlichster Wählergruppen Erfolge erzielen. Das zwingt sie zu weit reichenden Kompromissen in programmatischen Fragen, die soziale Bewegungen nicht

7 Lösche 2007; Bibby 2003; Maisel 2002; Klumpjan 1998. 
einzugehen bereit sind. Parteien sind vote maximizers, soziale Bewegungen dagegen policy maximizers (Berry 1997), die ihre Anliegen möglichst vollständig durchgesetzt sehen wollen. Für Parteien sind programmatische Kompromisse und eine additive Agenda, die die Anliegen der wichtigsten respektive artikuliertesten Wählergruppen bündelt, für den angestrebten Wahlsieg unerlässlich.

Ideologisch-puristische Kandidaten, die um der Reinheit ihrer Anliegen willen diese Strategie der Stimmenmaximierung ablehnen, haben selbst auf dem ticket der Demokratischen oder der Republikanischen Partei kaum eine Siegchance. Als unabhängige Kandidaten oder Vertreter einer Dritten Partei ist ihr Wahlkampf nachgerade aussichtslos: Das aus institutionellen, historischen und politisch-kulturellen Gründen bestehende Zweiparteiensystem errichtet hohe Hürden für die dauerhafte Etablierung einer Dritten Partei, zumindest auf Bundes- und Einzelstaatenebene, ${ }^{8}$ insbesondere dann, wenn sie in ideologischen Fragen kompromisslos auftritt. ${ }^{9}$ Seit der Entstehung der Republikanischen Partei (der „Grand Old Party“, G. O. P.) in der zweiten Hälfte des 19. Jahrhunderts konnte sich so auch keine neue Partei langfristig im politischen System der USA durchsetzen. Soziale Bewegungen haben insofern keinen Anreiz, eine eigene (dritte) Partei zu gründen. Entsprechend ist die Zahl der Interessengruppen, die eine soziale Bewegung ausbildet, in den Vereinigten Staaten sehr viel größer als in einem System, das die Formierung von Parteien prämiert, weil eine eigenständige (Bewegungs-)Partei die Anhänger und Ressourcen einer Bewegung stärker bindet.

\section{c) Infiltration von Parteien}

These 3: Soziale Bewegungen haben einen Anreiz, Parteien zu infiltrieren, um auf deren Kandidatenrekrutierung und Programmformulierung Einfluss zu nehmen.

Die Demokratische und die Republikanische Partei erfüllen trotz der eingetretenen Funktionsverluste bei der Vorbereitung und Durchführung von Wahlen weiterhin wichtige Aufgaben. So sind sie noch immer an der Auswahl des politischen Personals beteiligt, auch wenn durch die Einführung von Vorwahlen für beinahe alle politischen Ämter und Mandate in nahezu allen Bundesstaaten das letzte Wort über die Kandidatennominierung in die Hände der registrierten Wähler einer Partei (bzw. bei den selteneren open

8 Rosenstone/Behr/Lazarus 1996; Herrnson/Green 1997; Klumpjan 1998: 47 ff.

9 Erinnert sei an das Schicksal der verschiedenen sozialistischen Parteien in den USA. 
primaries: aller Wähler) gelegt worden ist. Obschon die Kandidatenauswahl kaum noch durch Parteiversammlungen (caucuses) erfolgt, haben die Parteien nach wie vor zahlreiche Möglichkeiten, zumindest indirekt die Elitenrekrutierung zu beeinflussen. Damit bleiben die Parteien die gate-keeper für den Zugang zu hohen und höchsten politischen Ämtern und Mandaten (Aldrich 1995: 273 f.; Burstein 1998: 46 f.).

Für soziale Bewegungen besteht daher ein Anreiz, die Demokratische oder die Republikanische Partei gezielt zu infiltrieren, um so über die $\mathrm{Zu}$ sammensetzung des politischen Führungspersonals mitzubestimmen und eigene oder ihnen ideologisch nahe stehende Kandidaten mit den Ressourcen der Partei unterstützen zu können (Sundquist 1983: 66). Darüber hinaus bietet die gezielte Infiltration der Parteikomitees die Chance, auf die Satzungen der Parteien Einfluss zu nehmen, die etwa den Modus der Delegiertenauswahl für die höchsten Beschlussfassungsgremien der Partei festlegen. Auf diesem Weg können auch die Parteiprogramme im Sinne der eigenen Agenda und ihrer zentralen „Prinzipien“ modifiziert werden. Dadurch vermag eine Bewegung das äußere Erscheinungsbild der Partei mit zu prägen und die Aufmerksamkeit der Öffentlichkeit auf ihre Themen zu lenken.

\section{d) Kooptation durch Parteien}

These 4: Die Parteien haben einen Anreiz, soziale Bewegungen zu kooptieren, um sich deren Anhänger als Wähler zu versichern.

Amerikanische Parteien sind breite Koalitionen aus sehr heterogenen Bevölkerungsgruppen, deren Zweck vor allem in der Nominierung und Durchsetzungen von Kandidaten für öffentliche Ämter liegt. Da sie in der stark segmentierten amerikanischen Gesellschaft nur mehrheitsfähig sind, wenn sie programmatisch flexibel und offen für neue politische Strömungen bleiben, gelten sie als „schwammig-aufsaugfähige“ Gebilde (Lösche 2007: 320). Parteieliten werden stets versuchen, neue soziale Bewegungen oder aufkommende Drittparteien der eigenen Wählerkoalition einzuverleiben, sobald sie eine kritische Größe erreichen. Eine solche Kooptation erfolgt dabei durch programmatische Annäherung (Berry/Schildkraut 1998). ${ }^{10}$ Sie wird dadurch erleichtert, dass die party platforms das Profil einer Partei zwar prägen, für die Amts- und Mandatsträger aber kaum primäre Richtschnur ihres

10 Eine solche Annäherung setzt allerdings voraus, dass eine zumindest grundsätzliche Kompatibilität des konkreten Forderungskatalogs einer Bewegung mit dem programmatischen Gesamtprofil einer Partei im relevanten Zeitraum besteht. 
Handelns sind. Durch die inhaltliche Berücksichtigung von Bewegungsthemen hofft eine Partei, Loyalitäten zu erzeugen, die die Unterstützer und Sympathisanten der Bewegung an sie binden. Nach deren „Eintritt“" soll die wiederholte Bestätigung der programmatischen Positionen ihre „Mitgliedschaft" konsolidieren helfen und verhindern, dass sie die „Exit"- oder „Voice“-Optionen (Hirschman 1970) wählen. Da die Kooptation einer ideologisch kontroversen sozialen Bewegung auch moderate Wählerstimmen kosten kann, legen die Parteieliten ihren Entscheidungen eine Gewinn- und Verlustrechnung der zu erwartenden Wählerwanderungen zugrunde. Doch auch im Falle einer negativen Entscheidung kann die Infiltration der Partei(-komitees) durch Anhänger einer Bewegung (vgl. These 3) kaum völlig verhindert werden, denn die locker strukturierten, organisatorisch wenig fixierten amerikanischen Parteien kennen keine formale Parteimitgliedschaft und daher auch keine Parteiausschlussverfahren. ${ }^{11}$

\section{Abbildung 1}

Soziale Bewegungen im politischen System der USA

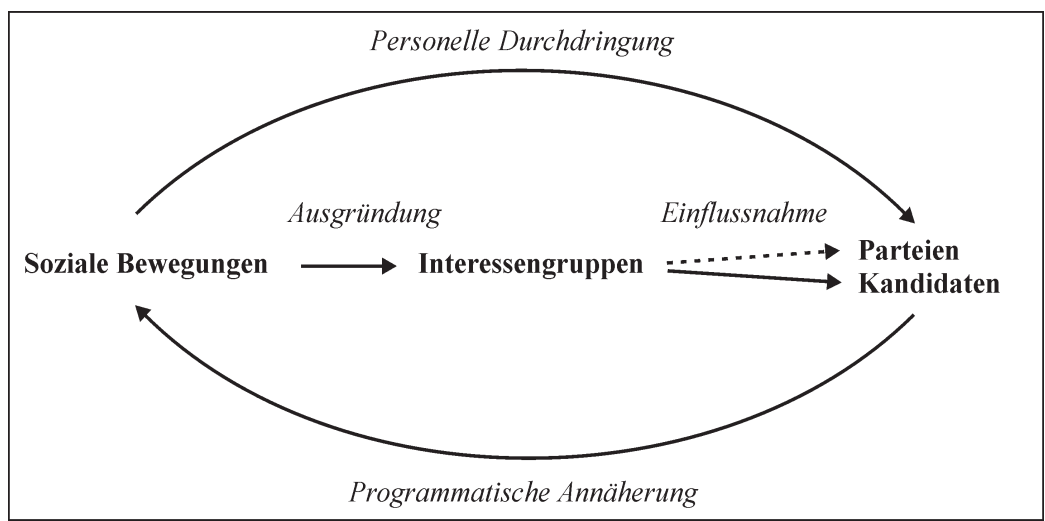

11 Als „Parteimitglied“ gilt in primary-Staaten, wer bei seiner Eintragung in das amtliche Wählerverzeichnis angibt, an den Vorwahlen einer bestimmten Partei teilnehmen zu wollen. Als „Parteimitglied“ kann auch angesehen werden, wer sich in Meinungsumfragen zu einer bestimmten Partei bekennt bzw. in Wahlen regelmäßig für eine bestimmte Partei entscheidet (,party in the electorate"). Dagegen gibt es in den USA keine durch Parteibuch und regelmäßige Beitragszahlung begründete Parteizugehörigkeit. 


\section{Die Thesen auf dem Prüfstand}

\section{a) Neue soziale Bewegungen}

Der Blick auf die Entwicklung der reformliberalen, sozial-emanzipatorischen Bewegungen der fünfziger bis siebziger Jahre des 20. Jahrhunderts bestätigt die genannten Thesen. Nach einer anfänglichen Phase der (Massen-)Mobilisierung von Unterstützern und Sympathisanten, in der mit bewegungsförmigen Protest-Aktivitäten (wie Demonstrationen, Boykotten, Sit-ins, gezielten Regelverstößen) die Aufmerksamkeit der Öffentlichkeit auf eine zunächst noch recht diffuse und übergreifende Agenda gelenkt werden sollte, bildeten die Bürgerrechts-, die Frauen-, Umwelt-, Friedens- und Homosexuellenbewegung allmählich Interessengruppen aus, die die gegebenen institutionellen Einflusskanäle des amerikanischen politischen Systems zunehmend professioneller nutzten, um ihren jetzt konkreter formulierten Forderungen Gehör zu verschaffen - was im Übrigen zu einem sprunghaften Anstieg der Zahl der public interest- bzw. single issue groups in den USA beitrug. ${ }^{12}$ Durch gezieltes Lobbying vor der Exekutive, der Legislative und - immer häufiger - den Gerichten sollten diese in wirkliche Reformpolitik umgesetzt werden.

Parallel zur organisatorischen Auskristallisierung der Bewegungen und der verstärkten Anwendung von Lobby-Strategien begannen ihre Anhänger mit der Infiltration der lokalen, einzelstaatlichen und nationalen Komitees der Demokratischen Partei. Diese öffnete sich durch programmatische (und innerorganisatorische) Zugeständnisse mehr und mehr den sozial-emanzipatorischen Bewegungen (Paulson 2000: 104 ff.), um sie in ihre seit der Zeit des New Deal weitgehend unverändert aus (gewerkschaftlich organisierten) Arbeitnehmern, Farmern, Katholiken, ethnischen Minderheiten und (weißen) Südstaatlern bestehende Wählerkoalition einzufügen (Burnham/ Chambers 1975; Sundquist 1983). Spätestens mit der Präsidentschaftskandidatur George McGoverns 1972 war dieser Prozess der Kooptation (und Infiltration) vollzogen: Die Demokratische Partei war zur „Partei der Bewegung“ geworden.

12 Zur Bürgerrechtsbewegung vgl. Morris 1984; Chong 1991; McAdam 1999; Kolb 2007: 97-189. Zur Frauenbewegung vgl. Rupp/Taylor 1987; Katzenstein/Mueller 1987; Staggenborg 1991; Ryan 1992; Costain 1981, 1992; Ferree/Martin 1995; Ferree/ Hess 2000. Zur Umwelt- und Anti-AKW-Bewegung vgl. Rucht 1994; Costain/Lester 1998; Shaiko 1999; Kolb 2007: 193-273. Zur Friedensbewegung vgl. Chatfield 1992; Meyer 1993; Small 2002. Zur Homosexuellenbewegung vgl. Cruikshank 1992; Adam 1995; Vaid 1995; Dufour 1998; Engel 2001; Rimmerman 2007. 
Anders als in Deutschland, wo sich die Umwelt-, Friedens- und Frauenbewegung Ende der siebziger, Anfang der achtziger Jahre zu einer eigenständigen Partei formierte („Die Grünen“), wählten die reformliberalen Bewegungen der USA den Weg der Interessengruppenbildung und der Infiltration einer bestehenden Partei, deren ideologisch-programmatisches Profil sich dadurch nach „links“ verschob. Diese Entscheidung war Ergebnis eines rationalen Nutzenkalküls der Bewegungseliten und Basisaktivisten, die vor dem Hintergrund der spezifischen politischen Chancenstruktur, d. h. der in den sechziger und siebziger Jahren gegebenen institutionellen, rechtlichen und politisch-kulturellen Rahmenbedingungen, die vorgefundenen Einflussmöglichkeiten immer konsequenter in ihren strategischen Planungen berücksichtigten. Innerhalb weniger Jahre gelang es ihnen auf diese Weise, weit reichende gesellschaftspolitische Reformen mit auf den Weg zu bringen. Die Beendigung der staatlichen Rassendiskriminierung und die Einführung von affirmative action-Programmen, die Wahlrechtsreformen der sechziger Jahre, die Frauenemanzipation und die Legalisierung der Abtreibung ebenso wie die gesetzlichen Maßnahmen zum Schutz von Homosexuellen vor Diskriminierung, die Verbesserung des Umwelt- und Verbraucherschutzes etc. können als Resultate des Wirkens der reformliberalen Bewegungen und der aus ihnen hervorgegangenen Interessengruppen betrachtet werden.

Insgesamt lässt sich somit eine zunehmende Etablierung der reformliberalen Kräfte im politischen System der USA seit den sechziger Jahren konstatieren. Selbst dort, wo wie etwa in Teilen der Bürgerrechtsbewegung weiter reichende Änderungsabsichten verfolgt wurden, kann von einer „Integration durch Partizipation" gesprochen werden.

\section{b) Die Christliche Rechte}

Untersuchungen zur „fundamentalistischen“ Protestbewegung der „Christlichen Rechten“, die Ende der siebziger Jahre als Reaktion auf die neuen sozialen Bewegungen entstand, ${ }^{13}$ zeigen, dass die genannten Thesen auch für sie Gültigkeit besitzen. Bei ihr lässt sich in den achtziger und neunziger Jahren ebenfalls eine Anpassung an die Funktionsimperative des politischen Systems der USA konstatieren. Betrachten wir dazu die Thesen im Einzelnen:

$\mathrm{Zu}$ These 1: Blickt man auf die Entwicklung der Christlichen Rechten in den achtziger und neunziger Jahren, so wird deutlich, dass die genannten push- und pull-Faktoren auch bei ihr in die erwartete Richtung gewirkt haben.

13 Brocker 2004; Brown 2002; Wilcox 2000; Minkenberg 1998; Watson 1997. 
Nach einer anfänglichen Phase des trial and error in Organisations- und Strategiefragen ist bei der Christlichen Rechten seit den späten achtziger Jahren ein deutlicher Trend zu verbandlichen Organisationsformen und Strategien erkennbar (Brocker 2004). Sie hat im Laufe der neunziger Jahre frühere Protestformen weitgehend durch professionelles Lobbying etc. ersetzt. Darüber hinaus weist sie kaum noch den für soziale Bewegungen typischen Netzwerk-Charakter auf. Sie stellt sich heute eher als ein Ensemble unabhängiger, religiös-politischer pressure groups dar. Ihre Organisationen verfügen gegenüber den frühen achtziger Jahren fast durchweg über einen stärker formalisierten Aufbau, geordnetere (hierarchisch-zentralisierte) Planungsstrukturen, festere Kommunikationswege, eine berechenbarere, langfristig angelegte Finanzierung und eine insgesamt größere Stabilität - all dies spezifische Merkmale eines Verbandes. Insofern ähneln Organisationen wie die „Christian Coalition" inzwischen eher amerikanischen Gewerkschaften wie den „United Auto Workers“ oder der „National Education Association“ (Gimpel 1998) als der kurzlebigen „Moral Majority“ der frühen achtziger Jahre. Diese Transformation hat die Strategiefähigkeit und die Durchschlagskraft der Christlichen Rechten deutlich gesteigert (vgl. These 1.2).

Den positiven Effekten stehen allerdings auch Kosten gegenüber: Die abnehmende Emphase der Anhängerschaft, das Ausscheiden der „Puristen“ aus der Bewegung, die die neue „Interessengruppenpolitik“ der Organisationen als ,politics as usual“ ablehnen, der Verlust von Unterstützern, die sich dem erkennbaren Macht- und Erwerbsinteresse der professionals verweigern, weil diese nach ihrer Auffassung bei der Verfolgung der ,ursprünglichen Bewegungsziele" zu viele Kompromisse eingehen, haben die Christliche Rechte an der Basis geschwächt. Mit der Institutionalisierung ging eine Art innere „Erstarrung“ einher, die den gegenwärtigen Zustand der Christlichen Rechten kennzeichnet.

Zu These 1.1: Im Fall der Christlichen Rechten existierten Ende der siebziger, Anfang der achtziger Jahre nur in der Frage des Abtreibungsrechts politische Organisationen (der Anti-Abtreibungsbewegung), die Bewegungsthemen aktiv vertraten. Obwohl die Zahl evangelikaler Christen in den Organisationen im Zuge der allgemeinen Politisierung des konservativ-protestantischen Milieus seit den späten siebziger Jahren zugenommen hatte, wurden sie von der Christlichen Rechten nicht gezielt infiltriert. Die formale Übernahme dieser Organisationen hätte den Exodus ihrer weiterhin mehrheitlich katholischen Anhängerschaft bewirkt, die zwar die Legalisierung des Schwangerschaftsabbruchs ablehnte, andere Ziele der Christlichen Rechten 
aber nicht teilte. Ihre Abwanderung hätte die Organisationen deutlich geschwächt. Daher konnte der Christlichen Rechten nicht an einer Eingemeindung der etablierten und teilweise äußerst schlagkräftigen pro-life-Gruppen gelegen sein. Punktuelle Kooperationen erschienen in diesem spezifischen inhaltlichen Kontext zweckmäßiger als die formale Übernahme oder der Aufbau eigener single issue groups.

Die Übernahme von Organisationen des Evangelikalismus und Fundamentalismus kam für die Christliche Rechte ebenfalls nicht in Betracht. Zwar machten sich auch bei diesen die Mobilisierungsbemühungen der Christlichen Rechten Ende der siebziger, Anfang der achtziger Jahre bemerkbar. So nahm der Einfluss von Fundamentalisten in der „Southern Baptist Convention", einer Dachorganisation baptistischer Kirchengemeinden, fortgesetzt zu. Die daraus resultierenden konservativeren Positionsbestimmungen der Organisation blieben jedoch weitgehend auf theologische (und allgemein moralische) Fragen beschränkt. Eine durchgängige Politisierung fand nicht statt; sie wäre ohnehin gegen die starke baptistische Tradition der Trennung von Religion und Politik nicht durchzusetzen gewesen (Kramnick/Moore 1997: 110-130) und hätte zur Spaltung dieser wie anderer kirchennaher Organisationen - etwa der neoevangelikalen „Christian Legal Society“ - geführt. Deshalb präferierte die Christliche Rechte hier wiederum nur punktuelle Kooperationen.

Angesichts der dargelegten Befunde kann der Fall der Christlichen Rechten die These 1.1 nicht bestätigen. Dass dennoch empirische Gründe für sie sprechen, zeigen Beispiele der reformliberalen, sozial-emanzipatorischen Bewegungen der sechziger und siebziger Jahre, die eine Reihe älterer, thematisch verwandter Verbände gezielt übernahmen: So wurde etwa der „Sierra Club", bis dato eine Art Alpenverein, von Anhängern der Umweltbewegung unterwandert und sukzessive in eine politische (ökologische) Interessengruppe transformiert (Lösche 2004: 364).

$\mathrm{Zu}$ These 1.2: Die intensivere Nutzung politischer Partizipationsmöglichkeiten auf allen Ebenen des politischen Systems - durch legislative lobbying und court lobbying, durch die Mitwirkung an lokalen Entscheidungsverfahren etc. - bewirkte eine Professionalisierung der Arbeit der Christlichen Rechten. Einige ihrer größten Erfolge waren erkennbar auf die verbesserte Handhabung bestimmter politischer Partizipationsformen wie direktdemokratischer Beteiligungsverfahren zurückzuführen: Das gilt etwa für die Aufhebung von Anti-Diskriminierungsmaßnahmen zum Schutz von Homosexuellen in Einzelstaaten und Kommunen, die Änderung einzelstaatlicher 
Verfassungen zum Verbot von „Homo-Ehen“ usw. durch Volksbegehren und Volksentscheid. Insofern findet These 1.2 - bezüglich des vermuteten $\mathrm{Zu}$ sammenhangs von Professionalisierung und erhöhter Durchschlagskraft (gemessen etwa an ihrem Einfluss auf das öffentliche agenda setting) - im Fall der Christlichen Rechten ihre Bestätigung. Damit geht allerdings nicht unmittelbar eine verbesserte Durchsetzungsfähigkeit oder Erfolgsquote von Organisationen einher: Der politische Erfolg hängt auch von zahlreichen Faktoren ab, die jenseits der Organisationen selbst liegen, ${ }^{14}$ hier aber nicht im Einzelnen diskutiert werden sollen. Lediglich auf die Rolle und Reaktion der jeweiligen Gegner ihrer Reformziele ist das Augenmerk zu richten. In dieser Hinsicht muss ergänzend eine weitere These aufgestellt werden:

These 1.3: Je durchschlagskräftiger aus sozialen Bewegungen hervorgegangene Interessengruppen werden und je umstrittener die von ihnen initiierten gesellschaftlichen und politischen Reformen sind, umso größer wird der Anreiz für oppositionelle Bewegungsunternehmer, ${ }^{15}$ die Gegner dieser Reformziele zu mobilisieren.

Die strukturelle Offenheit des politischen Systems, die konfliktfreudige politische Kultur und der partizipatorische Politikstil der USA (These 1) bieten Bewegungsunternehmern wiederum einen Anreiz zur verbandsförmigen Mobilisierung. Reformbefürworter und -gegner stehen sich dann in den verschiedenen Politikarenen mit jeweils eigenen Interessengruppen gegenüber. Die Christliche Rechte veranschaulicht dies auf zweifache Weise: Einerseits ist sie selbst als „Gegenbewegung“ zu bezeichnen, insofern sie als Reaktion auf die zunehmende Durchschlagskraft der reformliberalen, sozial-emanzipatorischen Bewegungen der sechziger und siebziger Jahre entstand. Andererseits veranlassten die anfänglichen Erfolge der Christlichen Rechten ihrerseits wiederum den Aufbau neuer (reform-)liberaler Organisationen. Auf die erfolgreiche Wählermobilisierung von „Moral Majority“ Ende der siebziger Jahre antworteten liberale Bewegungsunternehmer mit der Gründung von

14 Schon die These über den Zusammenhang von Professionalisierung und erhöhter Durchschlagskraft einer Organisation gilt nur unter der ceteris paribus-Klausel, d. h. bei gleich bleibender Ressourcenausstattung, insbesondere konstanter Anhängerschaft. Die Institutionalisierung von Bewegungsorganisationen, die die Professionalisierung ihrer Arbeit begleitet, scheint aber regelmäßig zum Ausscheiden bestimmter Anhängergruppen, der ,bewegten Puristen“, zu führen (vgl. These 1). Übersteigt deren Anteil einen kritischen Wert, mindert dies die Durchschlagskraft der Organisationen.

15 „The only way to defeat a social movement is with another social movement", formulierte ein Bewegungsunternehmer, der eine Organisation der Anti-Ökologiebewegung leitete; zitiert nach Berry 1997: 35. Vgl. auch Lo 1982; Zald/Useem 1987; Meyer/ Staggenborg 1996. 
Gegenorganisationen. Beispiele hierfür sind der Fernsehproduzent Norman Lear und sein „People for the American Way“ oder George McGoverns „Americans for Common Sense“ etc. Der Aufstieg der „Christian Coalition“ zur größten religiös-politischen pressure group der amerikanischen Politik Anfang der neunziger Jahre löste unmittelbar die Konstituierung religiöspolitischer Organisationen einer neuen „Christlichen Linken“ aus (wie „Call to Renewal“" und „Interfaith Alliance“).$^{16}$ Gegenorganisationen dieser Art konterkarieren die politischen Bemühungen selbst professionell arbeitender Gruppierungen. Die Resultante des jeweils ausgeübten Drucks und Gegendrucks ist deshalb nur am konkreten Einzelfall empirisch zu bemessen. Über die Auswirkung der durch Professionalisierung erzielten erhöhten Durchschlagskraft auf die Durchsetzungsfähigkeit und den politischen Erfolg einer Organisation kann insofern - nicht zuletzt aus diesem Grund - keine allgemeine Aussage gemacht werden.

$\mathrm{Zu}$ These 2: Dass für soziale Bewegungen in den USA kein Anreiz besteht, eine eigene Partei zu gründen, zeigen die reformliberalen, sozial-emanzipatorischen Bewegungen der sechziger und siebziger Jahre sehr deutlich. Sie verzichteten darauf, obwohl Ende der siebziger, Anfang der achtziger Jahre in vielen Ländern Europas „Grüne Parteien“ aus solchen Bewegungen hervorgingen (Müller-Rommel 1993). Zwar blieben deren Erfolge in den Vereinigten Staaten nicht unbemerkt. Trotz der erkennbaren Neigung von sozialen Bewegungen, Themen, Strategien und Taktiken anderer erfolgreicher in- wie ausländischer Bewegungen zu übernehmen (McAdam/Rucht 1993; Wolfe 1983), regten sie zur Nachahmung dennoch nicht an. ${ }^{17}$

Auch die religiös-politische Bewegung der Christlichen Rechten hat seit ihrer Entstehung keine Anstrengungen unternommen, sich als neue Partei zu konstituieren. Im Hinblick auf die geringen Erfolgsaussichten schienen die Kosten hierfür zu hoch. Als leichter und effizienter erwies es sich für sie wie für andere Bewegungen dagegen, eigene oder ideologisch nahe stehende Kandidaten auf dem ticket einer der beiden großen Parteien zu platzieren und

16 „Call to Renewal“ ist ein Aktionsbündnis liberaler und moderater Evangelikaler, „Interfaith Alliance“ eine Mitte der neunziger Jahre gegründete interkonfessionelle Gruppierung, die die Mobilisierungs- und Organisationstechniken der „Christian Coalition“" gezielt kopiert; vgl. Schwartz/Levine 1996: 233 ff.; Green 2000a: 10.

17 Die umwelt- und friedenspolitisch ausgerichtete „Citizens Party“, die 1980 den Umweltaktivisten Barry Commoner als Präsidentschaftskandidaten aufstellte, war ein äußerst kurzlebiger und erfolgloser Versuch, eine neue Partei in den USA zu etablieren. Ihr schlossen sich nur wenige Mitglieder der genannten Bewegungen an; die Mehrheit antizipierte die geringen Erfolgsaussichten dieser Partei und blieb ihr fern. 
mithilfe eigener Ressourcen, z. B. durch freiwillige Helfer, die Verteilung von voter guides etc., im Wahlkampf zu unterstützen (vgl. These 3). ${ }^{18}$

Durch den Verzicht auf eine Parteigründung wurde ein zusätzlicher Spielraum für die Bildung weiterer Policy-Organisationen eröffnet, die es erlaubten, das Feld der citizen- oder public interest groups auf allen Ebenen des politischen Systems noch stärker zu besetzen: Da die Ressourcen und Anhänger der Bewegung nicht von einer Partei absorbiert wurden, konnten politische Unternehmer und issue entrepreneurs um sie werben, mit ihrer Hilfe neue Organisationen aufbauen und neue „Produkte“ (Orientierungsleistungen und Policy-Positionen) auf dem politischen Markt platzieren. Untersuchungen zeigen, wie nach der erfolgreichen Gründung der ersten nationalen Organisationen der Christlichen Rechten Ende der siebziger Jahre in den neunziger Jahren mehr und mehr auf einzelne Regionen, Themen oder Strategien spezialisierte Gruppierungen ins Leben gerufen wurden, die sich durch Spenden, Mitgliedsbeiträge oder den Verkauf von Publikationen finanzierten (Brocker 2004).

$\mathrm{Zu}$ These 3: Wie die reformliberalen, sozial-emanzipatorischen Bewegungen seit den späten sechziger Jahren die Demokratische Partei infiltrierten, so begann auch die Christliche Rechte ab Mitte der achtziger Jahre die Republikanische Partei zu unterwandern, obwohl beide Bewegungen die Parteien zuvor wegen ihrer Korruptheit, „Untätigkeit“, politischen Ausrichtung und mangelnden Responsivität etc. heftig attackiert hatten. Anders jedoch als im Falle der reformliberalen Bewegungen war das Vorgehen der Christlichen Rechten nicht durch ,partizipatorische Ideale“ motiviert, sondern zunächst vom politischen Ehrgeiz eines Bewegungsunternehmers getrieben: Die eigene Präsidentschaftskandidatur im Blick hatte Pat Robertson seine Anhänger 1986/87 aufgefordert, sich auf den Parteiversammlungen der G. O. P. in die Gremien wählen zu lassen oder sich als Delegierte zu bewerben. Dadurch hoffte er, auf dem nationalen Parteikonvent genügend Unterstützung für seine Nominierung als Präsidentschaftskandidat zumindest im zweiten Wahlgang zu finden.

Zwar scheiterte Robertsons Plan, aber der ersten „Eintrittswelle“ evangelikaler Protestanten in die Republikanische Partei, die er maßgeblich mit

18 So verzichtete Marion „Pat“ Robertson, ein Fernsehprediger und Bewegungsunternehmer der Christlichen Rechten, nach seiner gescheiterten Kandidatur in den presidential primaries der G. O. P. 1988 bewusst auf die Gründung einer (christlichen) Partei, die er in einem politischen System mit anderen Charakteristika (etwa einem parlamentarischen System mit Mehrparteien- und Proporzwahlsystem) möglicherweise ins Leben gerufen hätte - und schuf statt dessen die „Christian Coalition“. 
initiiert hatte, folgten bald weitere, und so gelang es der Christlichen Rechten im Laufe der neunziger Jahre, erhebliches Gewicht in der Partei zu gewinnen (Brocker 2004: 191-215):

- Anhänger der Christlichen Rechten stellten in den neunziger Jahren, wie Untersuchungen von Persinos (1994), Clifton (2000) sowie Conger und Green (2002) belegen, in nicht wenigen counties und Bundesstaaten die Vorsitzenden der Parteikomitees. Vor allem Basis-Aktivisten, die durch ihre Tätigkeiten in den Organisationen der Christlichen Rechten längst zu „Daueraktivisten" und „Bewegungsprofis" geworden waren, engagierten sich auf diese Weise in der G. O. P.

- Unverkennbar trugen weite Passagen der Parteiprogramme auf Bundeswie Einzelstaatenebene vor allem im Süden die Handschrift der Christlichen Rechten, etwa dort, wo ein ausnahmsloses Verbot der Abtreibung gefordert, die Wiedereinführung des Schulgebets verlangt oder das „christliche Erbe“ des Landes betont wurde.

- Der Einfluss der Christlichen Rechten auf die Kandidatenauswahl wurde regelmäßig bei den Präsidentschaftswahlen offenbar, wo sie praktisch ein Vetorecht bei der Auswahl des Vizepräsidentschaftskandidaten hatte. Auch wenn ihr Kalkül, flankierend zu den Lobby-Bemühungen in den Parlamenten eigene Kandidaten auf dem ticket der Republikanischen Partei ins Rennen zu schicken, nicht aufging - unter anderem, weil sie die massive Gegenmobilisierung reformliberaler Organisationen provozierten -, bewährte sich die second-best-Strategie dennoch: Durch die Wahlkampfhilfe für ihr ideologisch nahe stehende Kandidaten der Republikanischen Partei konnte sie einer ganzen Reihe von äußerst konservativen Bewerbern (wie Fob James, David Beasley und Mike Huckabee, John Ashcroft und Tom DeLay) zu politischen Ämtern und Mandaten (wie Gouverneursposten, Sitzen im Kongress etc.) verhelfen (Rozell/Wilcox 1995, 1997; Green/Rozell/Wilcox 2000, 2003).

Das Gewicht, das die Christliche Rechte in den neunziger Jahren innerhalb der Gremien, bei der Formulierung der Wahlplattformen und bei der Kandidatenrekrutierung der Republikanischen Partei besaß, dokumentiert, wie erfolgreich die G. O. P. infiltriert worden und dadurch - zumindest im weiteren Sinne - zur „Partei der Bewegung“ (Minkenberg 1998: 341 ff.) geworden war.

Allerdings hatte der Nutzen, den die Christliche Rechte hieraus zog, auch seinen Preis. Die enge Verbindung zur Republikanischen Partei schränkte ihren Handlungsspielraum zunehmend ein: Die „exit"-Option (Hirschman 1970) stand ihr kaum noch zur Verfügung, die Drohung mit einem „Partei- 
austritt" zeigte bei den Parteieliten kaum noch Wirkung. Lediglich der Wechsel zur Demokratischen Partei, die sich in sozialmoralischen und religionspolitischen Fragen aber längst auf der ,progressiv“-liberalen Seite dieser Policy-Felder positioniert hatte, oder die Unterstützung einer Dritten Partei standen ihr als Alternativen noch offen. Das aber, und das war den Akteuren durchaus bewusst, wäre gleichbedeutend mit dem abrupten Sturz in die politische Bedeutungslosigkeit gewesen. ${ }^{19}$ Realistisch betrachtet, hatte die Christliche Rechte daher nur die Wahl zwischen zwei Handlungsoptionen: „voice“, bei deren Ausübung freilich die Gefahr bestand, der Öffentlichkeit das Bild einer zerstrittenen Partei zu präsentieren, und „loyalty“, die zunehmend von ihr auch eingefordert wurde.

Die hohen Investitionen der Christlichen Rechten in ihre „Parteimitgliedschaft", die Profil und Wählerschaft der G. O. P. durchaus verändert haben, stellen für sie selbst erhebliche barriers to exit dar. Sie wird sich auch weiterhin einem starken innerparteilichen Kompromissdruck ausgesetzt sehen, der sie zu beträchtlichen programmatischen Konzessionen zwingen dürfte, insbesondere dort, wo ihre Positionen mit wirtschaftlichen Interessen kollidieren (etwa in der Frage der biogenetischen Forschung oder der Handelsbeziehungen zu China). Ihm wird sie nachgeben müssen, will sie ihren Einfluss in der Partei nicht verlieren.

$\mathrm{Zu}$ These 4: Die Republikanische Partei unternahm seit 1980 gezielt den Versuch, die Christliche Rechte durch die Aufnahme ihrer zentralen Forderungen in das Parteiprogramm zu kooptieren. Der Kooptationsversuch der Parteieliten ging damit der dargestellten Infiltration der Partei durch die Christliche Rechte zeitlich deutlich voran. Insbesondere die Stellung der Partei zum Abtreibungsrecht wurde von deren Eliten systematisch den Positionen der neuen Bewegung angepasst. ${ }^{20}$ Große Teile der von der Christlichen Rechten mobilisierten evangelikalen Wähler, die bis in die achtziger Jahre hinein Demokratische Parteigänger gewesen waren oder zur Gruppe

19 So gab es für die Christliche Rechte auch keinen Anreiz, die „U. S. Taxpayers Party“ (die sich 1999 in „Constitution Party“ umbenannte) zu unterstützen, die von einem Aktivisten der Neuen Rechten, Howard Phillips, gegründet worden war: Zwar bemühte sich dieser um die Stimmen christlich-rechter Wähler, indem er etwa das radikale Steuersenkungsprogramm seiner Partei um sozialmoralische issues erweiterte, doch ohne Erfolg: Der Stimmenanteil der Partei blieb bei den Präsidentschaftswahlen von 1992 bis 2008 regelmäßig deutlich unter einem Fünftelprozent; vgl. Minkenberg 1998: 248 ff.; http://www.fec.gov/pubrec/2000presgeresults.htm; http://www.fec.gov/ pubrec/fe2004/2004pres.pdf; http://www.fec.goc/pubrec/fe2008/2008presgeresults.pdf (07.05.09).

20 Adams 1997; Sanbonmatsu 1998; Brocker 2004: 208 f. 
der Nichtwähler gehört hatten, konnten dadurch langfristig an die G. O. P. gebunden werden (Nesmith 1994; Green 2000b). Sie wurde zur „pro-life“Partei, so wie die Demokraten in den sechziger und siebziger Jahren durch ihre programmatische Annäherung an die neuen reformliberalen, sozialemanzipatorischen Bewegungen zur „pro-choice“-Partei geworden waren.

\section{Die Folgen für das Parteiensystem}

Betrachtet man die Dynamik, die die sozialen Bewegungen innerhalb und zwischen den Parteien ausgelöst haben, so kann mit Blick auf deren Folgen für das amerikanische Parteiensystem eine weitere, fünfte These aufgestellt werden:

These 5: Durch ihre parteipolitischen Aktivitäten bewirken soziale

Bewegungen eine wachsende ideologische Polarisierung im ameri-

kanischen Parteiensystem, die zu einer weiterreichenden Re-Konfi-

guration der bestehenden Wählerkoalitionen führt.

Vor dem Hintergrund der dritten und vierten These ist für die Beziehungen zwischen Parteien und sozialen Bewegungen im politischen System der USA folgende typische Interaktionsdynamik zu erwarten: Erreicht eine neue soziale Bewegung eine bestimmte kritische Größe, entsteht ein Anreiz für die Parteien, sie durch programmatische Annäherung zu kooptieren und sich damit ihrer Anhänger als Wähler zu versichern. Grundsätzlich könnten beide Parteien („A“ und „B“) einen solchen Versuch unternehmen, da die zentripetalen Kräfte des amerikanischen Zwei-Parteien-Systems programmatisch ähnliche, zur gedachten Mitte eines politisch-ideologischen Kontinuums hin orientierte Parteien erzeugen (Downs 1957: 115 ff.). In der Regel wird jedoch jene Partei die Kooptation betreiben, deren Programmatik im relevanten Zeitraum die größere Nähe zum konkreten Forderungskatalog einer neuen Bewegung aufweist. Um mögliche Wählerverluste durch die Änderung ihres programmatisch-ideologischen Profils zu vermeiden bzw. so niedrig wie möglich zu halten, wird es das Ziel der Partei sein, die Kooptation möglichst „ohne konkrete Gegenleistungen“ durchzuführen („cooptation but no benefits").

Gleichzeitig wirkt die spezifische Chancenstruktur des amerikanischen politischen Systems als Anreiz für soziale Bewegungen, Parteien zu infiltrieren, um auf deren Programmformulierung und Kandidatenrekrutierung Einfluss zu nehmen. Grundsätzlich kämen auch hierfür beide Parteien in Betracht, tatsächlich aber werden sie diejenige Partei auswählen, deren Eliten 
öffentlich Sympathie für ihre Themen signalisieren und sich programmatisch für ihren Forderungskatalog zu öffnen beginnen. Der Kooptation durch eine Partei (A) folgt ihre Infiltration durch die Bewegung.

Auf mittlere Sicht werden die Amts- und Mandatsträger der Partei A den Forderungen der Bewegung, die bald selbst zu einer neuen, ,innerparteilichen Strömung" avanciert sein wird, nachgeben müssen, um sich ihrer Wählerstimmen dauerhaft zu versichern. So werden sie deren Forderungen nicht nur regelmäßig in die Wahlprogramme der Partei aufnehmen, sondern auch nach erfolgreicher (Wieder-)Wahl kleinere Gegenleistungen für die erbrachte Wahlunterstützung - zunächst nur in symbolischer Form! - erbringen. Das aber wird die Bewegungsanhänger auf Dauer nicht zufrieden stellen: Damit wächst der Druck auf die Amts- und Mandatsinhaber, konkrete politische Maßnahmen zu ergreifen und die relevanten public policies im Sinne der Bewegung zu beeinflussen.

Das öffentliche Erscheinungsbild der Partei A wird sich im Zuge dieser Entwicklungen allmählich verändern und - im Falle einer liberalen sozialen Bewegung („LS“) - etwa nach „links“ verschieben (vgl. Abbildung 2). Dem Wählerstimmenzugewinn durch die Annäherung an die Bewegung stehen bald zunehmende Verluste in der „Mitte“ gegenüber. Drohen die Stimmenverluste die Zugewinne zu übersteigen, wird die Partei versuchen, den Prozess der Kooptation abzubrechen, indem sie etwa demonstrativ symbolisch bedeutsame Änderungen in ihrer party platform beschließt, d. h. eine programmatische Selbstdistanzierung vornimmt. Hat zu diesem Zeitpunkt jedoch die Infiltration der Partei(-komitees) durch Bewegungsaktivisten bereits begonnen und einen kritischen Wert überschritten, ist eine solche programmatische Moderierung und Selbstdistanzierung - gegen den Widerstand der „Parteibasis“ - kaum noch möglich. Parteieliten können gegen den konzentrierten Druck neuer Parteigänger keine wirksamen Gegenmaßnahmen ergreifen, da die locker strukturierten, organisatorisch wenig fixierten amerikanischen Parteien weder eine formale Mitgliedschaft noch ein Ausschlussverfahren kennen: Die „Geister, die sie rief“, wird sie nun nicht wieder los (,Zauberlehrling-Effekt").

Mit den Wählerstimmenverlusten der Partei in der „Mitte“ des politischideologischen Kontinuums entsteht ein Anreiz für die zweite Partei (B), sich programmatisch ebenfalls - von ihrem leicht „rechts“ von der „Mitte“ liegenden Ort aus - nach „links“ zu bewegen, um dort die Stimmen der enttäuschten Wähler der ersten Partei (A) einzusammeln. Dadurch droht die erste Partei strukturell in eine Minderheitsposition zu geraten. 
Nun werden sich die inzwischen wahrnehmbaren Erfolge der Bewegung LS, die sich an ihrem Einfluss auf die Gestaltung bestimmter Policies, die Programmformulierung und Kandidatenrekrutierung der Partei A sowie - indirekt - auf die Programmentwicklung der zweiten Partei B zeigen, auch im Bewegungssektor selbst bemerkbar machen und dort Gegenkräfte provozieren. Denn je durchschlagskräftiger Bewegungen werden - so These 1.3 - und je umstrittener die von ihnen initiierten gesellschaftlichen und politischen Reformen sind, umso größer wird der Anreiz für oppositionelle Bewegungsunternehmer, die Gegner dieser Reformziele zu mobilisieren.

Gelingt die Mobilisierung einer solchen „Gegen-Bewegung“ („KS“) und erreicht sie eine kritische Größe, dann entsteht für die zweite Partei (B) ein Anreiz, die neue Bewegung durch programmatische Annäherung zu kooptieren. Da sie die Stimmenzugewinne, die sie durch die gezielt initiierte „Linksbewegung“ in der "Mitte“ - zu Lasten der Partei A - errungen hat, nicht gefährden will, wird auch ihr an einer „Kooptation (von KS) ohne wirkliche Gegenleistungen" gelegen sein.

Aufgrund der spezifischen Chancenstruktur des amerikanischen politischen Systems hat nun aber auch die neue (Gegen-)Bewegung KS ein vitales Interesse daran, eine Partei zu infiltrieren, um auf deren Programmformulierung und Kandidatenrekrutierung Einfluss zu nehmen. Für sie kommt hierfür - wegen der inzwischen erfolgten ideologisch-programmatischen Ausrichtung der Partei A entlang der neuen Konfliktlinie - nur die zweite Partei in Betracht, wobei deren Kooptationsversuche einen zusätzlichen Anreiz bieten. Die Kooptationsversuche beenden die programmatische „Linksbewegung" von B. Die einsetzende Infiltration der Parteikomitees durch die Aktivisten der Gegen-Bewegung KS wird zu einem zusätzlichen „Rechtsruck“ führen: Selbst wenn hierdurch massive Stimmenverluste in der „Mitte“ ausgelöst werden sollten (die starke „Links“-Bewegung der Partei A wird diese Verluste in Grenzen halten), könnten die Parteieliten diese Entwicklung nun nicht mehr verhindern, da die fortschreitende Unterwanderung der Partei(-komitees) jede programmatische Moderierung und Selbstdistanzierung der Partei B von der Gegen-Bewegung KS unmöglich machen wird. Auch die Eliten der zweiten Partei werden so Opfer des oben geschilderten „ZauberlehrlingEffekts": Die Bewegungsanhänger, die sie selbst gerufen haben, werden Partei B weiter nach „rechts“ schieben, als den Parteieliten um ihrer Wahlerfolge willen lieb sein kann. Soziale Bewegungen und Gegen-Bewegungen bewirken so eine ideologische Polarisierung innerhalb des amerikanischen 
Parteiensystems, die mit einer entsprechenden Re-Konfiguration der Wählerkoalitionen einhergeht.

\section{Abbildung 2}

Ideologische Ausrichtung amerikanischer Parteien und der Einfluss sozialer Bewegungen

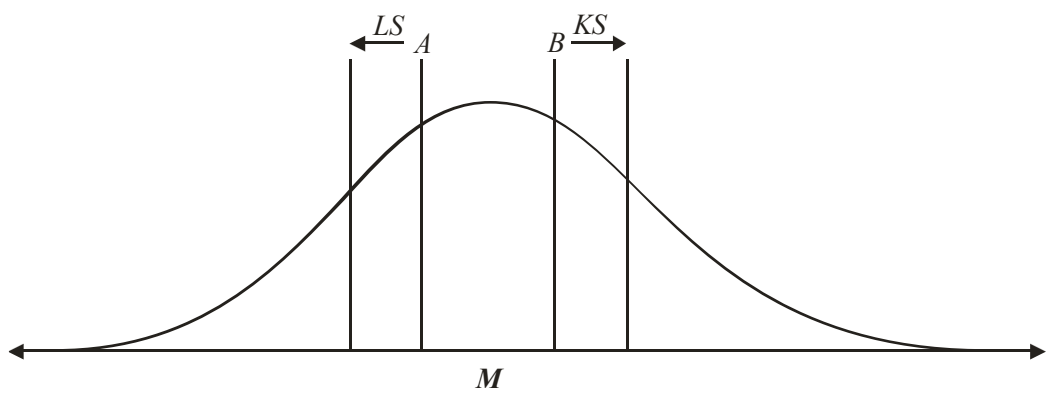

M: Mitte des politisch-ideologischen Spektrums, ideologische Ausrichtung des Bevölkerungsdurchschnitts. A: Partei „A“; B: Partei „B“; LS: Liberale soziale Bewegung; KS: Konservative soziale Bewegung

Auf lange Sicht werden sich jedoch die zentripetalen Kräfte des Parteiensystems wieder durchsetzen. Als Katalysator kann das Entstehen einer Dritten Partei oder die Präsidentschaftskandidatur eines unabhängigen dritten Kandidaten wirken. Doch auch ohne einen solchen externen Auslöser werden sich ausreichend starke Kräfte entwickeln, die beide Parteien erneut in die „Mitte“ des politisch-ideologischen Kontinuums zwingen: Schon durch die „Links"-Bewegung von Partei B, die Partei A strukturell in eine Minderheitsposition brachte, entstand für diese ein Anreiz, sich programmatisch wieder zur „Mitte“ hin zu orientieren. Ihre neue innerparteiliche Strömung (LS) wird dies zunächst zu verhindern suchen.

Mit der einsetzenden „Rechts-Bewegung“ der zweiten Partei (B), ausgelöst durch die Aktivitäten der Gegen-Bewegung (KS), wird der Druck auf die Bewegungsfraktion LS der Partei A jedoch derart stark zunehmen, dass sie die programmatischen Re-Orientierungsbemühungen der Parteieliten nicht länger verhindern können wird. Sobald Kandidaten der ersten Partei (A) in den Vor- und Hauptwahlen erfolgreich sind, die ihren Wahlkampf ohne 
Rücksicht auf die Ziele der Bewegung (LS) bestritten haben, werden sich auch die Bewegungsaktivisten einer Moderierung des Parteiprofils nicht länger widersetzen können. Wollen sie ihren Einfluss nicht völlig verlieren, werden sie den Weg der Partei zurück in die „politische Mitte“ mitgehen müssen. Nach der programmatisch-ideologischen Neu-Ausrichtung ,ihrer" eigenen Partei (B) wird die „rechte“ Gegen-Bewegung die Moderierung des programmatischen Profils der ersten Partei (A) zunächst als weiteren Erfolg ihrer Aktivitäten verbuchen!

Doch genau diese „Erfolge“ werden nun ihrerseits die zweite Partei (B) strukturell in eine Minderheitsposition bringen. Denn aufgrund der politischideologischen Neu-Positionierung beider Parteien erhöhen sich die Erfolgschancen der ersten Partei (A) und ihrer Kandidaten signifikant. Die Moderierung ihres programmatischen Profils wird nun immer häufiger Wahlerfolge nach sich ziehen. Damit steigt zugleich der Druck auf die zweite Partei (B), sich erneut zur „politischen Mitte“ hin zu bewegen. Eine ganze Reihe von zermürbenden Wahlniederlagen und erste erfolgreiche Kandidaturen an den politischen Zielen der Gegen-Bewegung (KS) vorbei werden daher bald auch sie wieder in die „Mitte“ rücken lassen. Die zentripetalen Kräfte des Parteiensystems setzen sich durch. Sie zwingen am Ende beide - die „linke“ wie die „rechte“ Bewegung bzw. Gegen-Bewegung und die aus ihnen hervorgegangenen Partei-Fraktionen - zu einer Moderierung ihrer issue agenda.

Die politischen Entwicklungen seit den sechziger Jahren veranschaulichen sehr deutlich die Wirksamkeit dieser Mechanismen: Die Demokratische Partei versuchte seit den frühen sechziger Jahren zunächst die Bürgerrechtsbewegung, später auch andere reformliberale, sozial-emanzipatorische Bewegungen (wie die Frauen-, die Umwelt- und die Homosexuellenbewegung) durch programmatische Annäherung zu kooptieren. Dem Wählerstimmenzugewinn, den sie dadurch - vor allem in den Staaten des Nordostens und Westens - zu verzeichnen hatte, standen jedoch zunehmend Stimmenverluste unter Stammwählergruppen, wie weißen Südstaatlern und traditionalistischen Katholiken, gegenüber (Ginsberg/Shefter 1985). Versuche, sich daraufhin von den linksliberalen Bewegungen programmatisch wieder zu distanzieren, schlugen jedoch fehl: Deren Anhänger hatten die Demokratische Partei Anfang der siebziger Jahre bereits soweit infiltriert, dass sie maßgeblich auf die Parteiorganisation, -statuten und -programmatik Einfluss nehmen konnten. Die „Geister“, die die Partei - anfangs gezielt - „gerufen“ hatte, wurde sie nun nicht mehr los. 
Als 1972 der Kandidat des „linken“ Flügels der Partei, George McGovern, für die Präsidentschaftswahl nominiert wurde und eine schwere Wahlniederlage gegen Richard Nixon einstecken musste, zeigte sich, dass die Demokratische Partei aufgrund ihrer ideologisch-programmatischen Neuausrichtung in eine strukturelle Minderheitsposition zu geraten drohte (Lawrence 1997). Seit Anfang der siebziger Jahre signalisierten Umfragen immer deutlicher einen Trend der Wähler hin zur Republikanischen Partei (Petrocik 1981; Campbell/Sumners 1990). Auch wenn die Auswirkungen der „WatergateAffäre" zunächst weitere schwere Niederlagen für die Demokratische Partei auf Bundesebene verhinderten, brachten die Wahlen der achtziger Jahre erneut Stimmenverluste. Seither bemühten sich die Eliten (insbesondere das „Democratic Leadership Council“) nachhaltig um eine Moderierung des programmatisch-ideologischen Profils ihrer Partei und damit einer Rückkehr in die ,politische Mitte“ (Edsall/Edsall 1991; Lind 1996: 56 ff.).

Opfer eines ähnlichen „Zauberlehrling-Effekts“ wird seit den neunziger Jahren auch die Republikanische Partei. Seit 1980 hatte sie zunächst gezielt und erfolgreich eine Strategie der Kooptation der Christlichen Rechten betrieben. Sie hatte sozialmoralische und religionspolitische Themen in ihre platforms aufgenommen, um die evangelikalen Anhänger der Bewegung als Wähler zu gewinnen, ohne allerdings an eine wirksame Umsetzung ihrer Forderungen zu denken. Präsident Reagan etwa unternahm so gut wie keine Anstrengungen, Verfassungsergänzungen zum Verbot der Abtreibung oder zur Wiedereinführung des Schulgebets zum Erfolg zu verhelfen (Brocker 2004: 106-110). Die Strategie der „,cooptation without benefits“ schien zunächst aufzugehen: 1984 wurde Reagan mit großer Mehrheit, auch der großen Mehrheit der Stimmen evangelikaler Protestanten, wiedergewählt. Offenbar war es ihm und der Republikanischen Partei gelungen, die Anhänger der Christlichen Rechten durch symbolische Politik zufrieden zu stellen und sie in die eigene Wählerkoalition zu integrieren, ohne andere Wählergruppen abzuschrecken.

Bereits Ende der achtziger Jahre zeichnete sich jedoch ab, dass das neue konservativ-protestantische Image der Partei auf Dauer auch Stimmen kosten würde. Vor allem pro-choice-Wähler, die die strikt ablehnende Haltung der G. O. P. in der Frage des Schwangerschaftsabbruchs nicht teilten, und libertarians, die alle Eingriffe des Staates in die Privatsphäre der Bürger ablehnten, unabhängig davon, ob sie wirtschaftspolitisch oder sozialmoralisch motiviert waren, begannen sich von der Partei abzuwenden. Ein erster Vorstoß der Parteispitze, das Image der G. O. P. zu moderieren und durch eine 
demonstrative Änderung der Position zum Abtreibungsrecht eine Selbstdistanzierung von der Christlichen Rechten herbeizuführen, scheiterte am Widerstand der Bewegungsaktivisten, die die Partei 1992 bereits soweit kontrollierten, dass auf dem Parteikonvent in Houston keine Neupositionierung in dieser Frage durchgesetzt werden konnte.

Die Aktivitäten der Bewegung verschoben das ideologisch-programmatische Profil der Republikanischen Partei vielmehr weiter nach „rechts“. Mit der Niederlage George H. W. Bushs in der Präsidentschaftswahl 1992, in der nicht wenige Stammwähler der G. O. P. für den Demokratischen Bewerber Bill Clinton oder für den unabhängigen Kandidaten Ross Perot votierten, der eine große Zahl von libertarians für sich zu mobilisieren verstand, ${ }^{21}$ wurde offenbar, dass sich ein partielles realignment entlang einer neuen, sozialmoralischen Konfliktlinie vollzogen hatte, das der Republikanischen Partei auch in Zukunft Stimmenverluste bescheren würde. Dadurch drohte sie - zumindest auf Bundesebene - strukturell in eine Minderheitsposition zu geraten. Versuche der Parteieliten (insbesondere der „Republican Majority Coalition"), eine erneute Wahlniederlage durch die inhaltliche Distanzierung von der Christlichen Rechten abzuwenden, schlugen jedoch vier Jahre später ebenfalls fehl: Die Zahl der christlich-rechten Parteitagsdelegierten hatte 1996 gegenüber 1992 noch einmal zugenommen. Gegen ihren Widerstand ließ sich auf dem Nominierungskonvent in San Diego eine demonstrative Änderung der abortion plank der party platform nicht durchsetzen. Am Ende wurde ein Wahlprogramm verabschiedet, in dem die Position des eigenen Präsidentschaftskandidaten zum Abtreibungsrecht als Minderheitsvotum nur im Appendix erschien. Bob Dole verlor die Wahl gegen den Demokratischen Amtsinhaber Bill Clinton, der das politisch-ideologische Profil seiner eigenen Partei inzwischen weitgehend moderiert hatte und sich selbst als middleof-the-road und „New Democrat" präsentierte ${ }^{22}$ (Bibby 2003: $143 \mathrm{ff}$.).

Die Niederlagen der G. O. P. in den Präsidentschaftswahlen von 1992 und 1996 sowie die wiederholten Mandatsverluste im Kongress zwischen 1996 und 2000 bzw. 2006 (Jäger/Haas/Welz 2007: 519) führten bei den Bewegungseliten der Christlichen Rechten zu einem Umdenken, wie es sich ganz ähnlich auf reformliberaler Seite in den achtziger Jahren vollzogen hatte: Weil sie ihr Schicksal derart eng mit einer der beiden Parteien verbunden

21 Gold 1995; Abramson u. a. 1995; Decker 2000: 139-157.

22 Clinton bewegte sich während seiner Amtszeit innenpolitisch weiter nach „rechts“ und übernahm nicht wenige Forderungen der Konservativen (Abbau des Haushaltsdefizits, Re-Föderalisierung der Sozialpolitik und Re-Moralisierung der persönlichen Existenzsicherung durch Unterzeichnung der Welfare Reform Bill etc.). 
hatten, musste ihnen an einer Verbesserung der Wahlchancen dieser Partei gelegen sein. Denn nur dann würden sie weiterhin Einfluss auf die politischen Entscheidungsprozesse nehmen können. Zudem würden ihre eigenen Organisationen nur dauerhaften Bestand haben, wenn sie politische Erfolge vorzuweisen hatten. Nicht zuletzt also um ihre eigene Machtbasis zu erhalten, mussten die Bewegungseliten den Weg der programmatischen Moderierung der Republikanischen Partei mitgehen: Die Zurückhaltung der Christlichen Rechten in den Präsidentschaftswahlen zu Beginn des dritten Milleniums und die Tatsache, dass die Parteieliten eine - wenn auch vorerst noch minimale - programmatische Re-Orientierung zur ,politischen Mitte“ innerparteilich durchsetzen konnten, ${ }^{23}$ belegen die Folgen solcher Überlegungen.

\section{Schluss}

Die dargestellten Interaktionsprozesse und Entwicklungsdynamiken im intermediären System der USA dokumentieren die zyklische Natur der amerikanischen Politik. Auf eine Phase der ideologischen Polarisierung der Parteien folgt eine Periode der Mäßigung. Denn auf lange Sicht zwingen die zentripetalen Kräfte des politischen Systems selbst jene Parteifraktionen, die aus sozialen Bewegungen hervorgegangen sind, zu einer Moderierung ihrer issue agenda. Der Druck der Christlichen Rechten auf die G. O. P. - diese Prognose lässt sich nach dem Gesagten wagen - wird weiter nachlassen und das politisch-ideologische Profil der Partei in Zukunft moderiert werden - ähnlich wie es bei der Demokratischen Partei im Laufe der achtziger und neunziger Jahre der Fall war.

Sobald diese Re-Orientierung der G. O. P. innerparteilich durchgesetzt ist - und die Anzeichen hierfür mehren sich nach der Niederlage in den Präsidentschafts- und Kongresswahlen von 2008 -, wird der politische Zyklus, der mit der Mobilisierung der neuen sozialen Bewegungen in den sechziger Jahren begann, abgeschlossen sein.

23 Wieland 2000; Piegsa 2008; Barbour touts moderate McCain, in: The Washington Times 29.04.08. 


\section{Literatur}

Abramson, Paul R./Aldrich, John H./Paolino, Phil/Rohde, David W., 1995:

Third-Party and Independent Candidates in American Politics: Wallace, Anderson and Perot, in: Political Science Quarterly 110, 349-368.

Adam, Barry D., 1995: The Rise of a Gay and Lesbian Movement, Boston (Revised Edition).

Adams, Greg D., 1997: Abortion: Evidence of an Issue Evolution, in: American Journal of Political Science 41 (3), 718-737.

Aldrich, John H., 1995: Why Parties? The Origin and Transformation of Party

Politics in America, Chicago/London.

Berry, Jeffrey M., 1997: The Interest Group Society, New York (3. Aufl.).

Berry, Jeffrey M./Schildkraut, Deborah, 1998: Citizen Groups, Political Par-

ties, and Electoral Coalitions, in: Costain/McFarland 1998,136-156.

Bibby, John F. (Hrsg.), 2003: Politics, Parties, and Elections in America, Belmont (5. Aufl.).

Brocker, Manfred, 2004: Protest - Anpassung - Etablierung. Die Christliche Rechte im politischen System der USA, Frankfurt a. M./New York.

Brown, Ruth Murray, 2002: For a „Christian America“. A History of the Religious Right, Amherst.

Burnham, Walter Dean/Chambers, William N. (Hrsg.), 1975: The American

Party Systems. Stages of Political Development, New York/Oxford.

Burstein, Paul, 1998: Interest Organizations, Political Parties, and the Study of Democratic Politics, in: Costain/McFarland 1998, 39-56.

Caldeira, Gregory A./Wright, John R., 1998: Organized Interests before the Supreme Court: Paper presented at the Annual Meeting of the American Political Science Association, Boston.

Campbell, James E./Sumners, Joe A., 1990: Presidential Coattails in Senate Elections, in: American Political Science Review 84 (2), 513-524.

Chatfield, Charles, 1992: The American Peace Movement. Ideals and Activism, New York.

Chong, Dennis, 1991: Collective Action and the Civil Rights Movement, Chicago/London.

Clifton, Brett M., 2000: Mobilization, Message, or Money? Christian Coalition Strategies of Influence in the Republican Party. Paper presented at the 58th Annual Meeting of the Midwest Political Science Association, Chicago. 
Conger, Kimberly H./Green, John C., 2002: Spreading Out and Digging In. Christian Conservatives and State Republican Parties, in: Campaigns and Elections 23, February, 58-65.

Costain, Anne N., 1981: Representing Women: The Transition from Social Movement to Interest Group, in: Western Political Science Quarterly 34 (1), 100-113.

Costain, Anne N., 1992: Inviting Women's Rebellion: A Political Process Interpretation of the Women's Movement, Baltimore/London.

Costain, Anne N./McFarland, Andrew S. (Hrsg.), 1998: Social Movements and American Political Institutions. Lanham.

Costain, W. Douglas/Lester, James P., 1998: The Environmental Movement and Congress, in: Costain/McFarland 1998, 185-198.

Cruikshank, Margaret, 1992: The Gay and Lesbian Liberation Movement, New York.

Decker, Frank, 2000: Parteien unter Druck. Der neue Rechtspopulismus in den westlichen Demokratien, Opladen.

Downs, Anthony, 1957: An Economic Theory of Democracy, New York.

Dufour, Claude, 1998: Mobilizing Gay Activists, in: Costain/McFarland 1998, 59-72.

Edsall, Thomas B./Edsall, Mary D., 1991: Chain Reaction: The Impact of Race, Rights, and Taxes on American Politics, New York.

Engel, Stephen M., 2001: The Unfinished Revolution. Social Movement Theory and the Gay and Lesbian Movement, Cambridge.

Ferree, Myra M./Martin, Patricia Y. (Hrsg.), 1995: Feminist Organizations.

Harvest of the New Women's Movement, Philadelphia.

Ferree, Myra M./Hess, Beth B., 2000: Controversy and Coalition. The New Feminist Movement across four Decades of Change, New York (3. Aufl.).

Freeman, Jo/Johnson, Victoria (Hrsg.), 1999: Waves of Protest. Social Movements Since the Sixties, Lanham.

Gimpel, James G., 1998: Grassroots Organizations and Equilibrium Cycles in Group Mobilization and Access, in: Paul S. Herrnson/Ronald G.Shaiko/ Clyde Wilcox (Hrsg.), The Interest Group Connection. Electioneering, Lobbying, and Policymaking in Washington, Chatham, 100-115.

Ginsberg, Benjamin/Shefter, Martin, 1985: A Critical Realignment? The New Politics, the Reconstituted Right, and the 1984 Election, in: Michael

Nelson (Hrsg.), The Elections of 1984, Washington DC, 1-25.

Gold, Howard J., 1995: Third Party Voting in Presidential Elections: A Study of

Perot, Anderson, and Wallace, in: Political Research Quarterly 48, 751-773. 
Goldstone, Jack A. (Hrsg.), 2003: States, Parties, and Social Movements, Cambridge.

Green, John C., 2000a: The Christian Right and the 1998 Elections: An Overview, in: ders./Mark J. Rozell/Clyde Wilcox (Hrsg.), Prayers in the Precincts. The Christian Right in the 1998 Elections, Washington DC, 1-19. Green, John C., 2000b: Religion and Politics in the 1990s: Confrontations and Coalitions, in: Mark Silk (Hrsg.), Religion and American Politics. The 2000 Election in Context, Hartford, 19-40.

Green, John C./Rozell, Mark J./Wilcox, Clyde (Hrsg.), 2000: Prayers in the

Precincts: The Christian Right in the 1998 Elections, Washington DC.

Green, John C./Rozell, Mark J./Wilcox, Clyde (Hrsg.), 2003: The Christian

Right in American Politics. Marching to the Millennium, Washington DC. Heclo, Hugh, 1978: Issue Networks and the Executive Establishment, in: Anthony King (Hrsg.), The New American Political System, Washington DC, 87-124.

Herrnson, Paul S./Green, John C. (Hrsg.), 1997: Multiparty Politics in America, Lanham.

Hirschman, Albert O., 1970: Exit, Voice, and Loyalty, Cambridge.

Imig, Douglas R., 1998: American Social Movements and Presidential Administrations, in: Costain/McFarland 1998, 159-170.

Jäger, Wolfgang/Haas, Christoph M./Welz, Wolfgang (Hrsg.), 2007: Regierungssystem der USA, München/Wien (3. Aufl.).

Katzenstein, Mary F./Mueller, Carol M. (Hrsg.), 1987: The Women's Movements of the United States and Western Europe: Consciousness, Political Opportunity, and Public Policy, Philadelphia.

Klein, Ansgar/Legrand, Hans-Josef/Leif, Thomas (Hrsg.), 1999: Neue Soziale Bewegungen. Impulse, Bilanzen und Perspektiven, Opladen/Wiesbaden. Klumpjan, Helmut, 1998: Die amerikanischen Parteien. Von ihren Anfängen bis zur Gegenwart, Opladen.

Kolb, Felix, 2007: Protest and Opportunities. The Political Outcomes of Social Movements, Frankfurt/New York.

Kramnick, Isaac/Moore, R. Laurence, 1997: The Godless Constitution. The Case against Religious Correctness, New York/London.

Lawrence, David G., 1997: The Collapse of the Democratic Presidential Majority: Realignment, Dealignment and Electoral Change from Franklin Roosevelt to Bill Clinton, Boulder.

Lind, Michael, 1996: Up From Conservatism. New York. 
Lo, Clarence Y. H., 1982: Countermovements and Conservative Movements in the Contemporary U. S., in: Annual Review of Sociology 8, 107-134.

Lösche, Peter, 1989: Amerika in Perspektive. Politik und Gesellschaft der Vereinigten Staaten, Darmstadt.

Lösche, Peter, 2004: Verbände, Gewerkschaften und das System der Arbeitsbeziehungen, in: ders./Hans Dietrich von Loeffelholz (Hrsg.), Länderbericht USA, Bonn (4. Aufl.), 353-389.

Lösche, Peter, 2007: Die politischen Parteien, in: Wolfgang Jäger/Christoph M. Haas/Wolfgang Welz (Hrsg.), Regierungssystem der USA, München/ Wien (3. Aufl.), 289-325.

Lowi, Theodore J./Ginsberg, Benjamin/Shepsle, Kenneth A., 2006: American Government. Power and Purpose, New York/London (9. Aufl.).

Maisel, L. Sandy (Hrsg.), 2002: The Parties Respond. Changes in American Parties and Campaigns, Boulder (4. Aufl.).

McAdam, Doug, 1996: Conceptual Origins, Current Problems, Future Directions, in: ders./John D. McCarthy/Mayer N. Zald (Hrsg.), Comparative Perspectives on Social Movements. Political Opportunities, Mobilizing Structures, and Cultural Framings, Cambridge, 23-40.

McAdam, Doug, 1999: Political Process and the Development of Black Insurgency, 1930-1970, Chicago/London (2. Aufl.).

McAdam, Doug/McCarthy, John D./Zald, Mayer N. (Hrsg.), 1996: Comparative Perspectives on Social Movements. Political Opportunities, Mobilizing Structures, and Cultural Framings, Cambridge.

McAdam, Doug/Rucht, Dieter, 1993: The Cross-National Diffusion of Movement Ideas, in: Annals of the American Academy of Political and Social Science 528, 56-74.

McCarthy, John D./Britt, David W./Wolfson, Mark, 1991: The Institutional Channeling of Social Movements by the State in the United States, in: Research in Social Movements, Conflict and Change 13, 45-76.

Meyer, David S., 1993: Protest Cycles and Political Process: American Peace Movements in the Nuclear Age, in: Political Research Quarterly 46, 451-479.

Meyer, David S./Jenness, Valerie/Ingram, Helen (Hrsg.), 2005: Routing the Opposition. Social Movements, Public Policy, and Democracy, Minneapolis.

Meyer, David S./Staggenborg, Suzanne, 1996: Movements, Countermovements, and the Structure of Political Opportunity, in: American Journal of Sociology 101 (6), 1628-1660. 
Meyer, David S./Tarrow, Sidney (Hrsg.), 1998: The Social Movement Society. Contentious Politics for a New Century, Lanham.

Minkenberg, Michael, 1998: Die neue radikale Rechte im Vergleich. USA, Frankreich, Deutschland, Opladen/Wiesbaden.

Morris, Aldon D., 1984: The Origins of the Civil Rights Movement. Black Communities Organizing for Change, New York.

Morris, Aldon D./Mueller, Carol M. (Hrsg.), 1992: Frontiers in Social Movement Theory, New Haven/London.

Müller-Rommel, Ferdinand, 1993: Grüne Parteien in Westeuropa. Entwicklungsphasen und Erfolgsbedingungen, Opladen.

Nedelmann, Birgitta, 1995: Soziale Bewegungen, in: Wolfgang Jäger/ Wolfgang Welz (Hrsg.), Regierungssystem der USA. Lehr- und Handbuch, München/Wien, 330-342.

Nesmith, Bruce, 1994: The New Republican Coalition. The Reagan Campaigns and White Evangelicals, New York.

Paulson, Arthur, 2000: Realignment and Party Renewal. Understanding American Electoral Politics at the Turn of the Twenty-First Century, Westport.

Persinos, John F., 1994: Has the Christian Right Taken Over the Republican Party?, in: Campaigns and Elections 15, September, 20-24.

Petrocik, John R., 1981: Party Coalitions. Realignment and the Decline of the New Deal Party System, Chicago.

Piegsa, Oskar, 2008: Kater und Kopfschmerz. Schlechte Stimmung bei Amerikas Konservativen. Der republikanische Spitzenkandidat McCain ist ihnen zu moderat, in: Die ZEIT online, 10. Februar.

Rimmerman, Craig A., 2007: The Lesbian and Gay Movements: Assimilation or Liberation?, Boulder.

Rosenstone, Steven J./Behr, Roy L./Lazarus, Edward H., 1996: Third Parties in America. Citizen Response to Major Party Failure, Princeton (2. Aufl.).

Rozell, Mark J./Wilcox, Clyde (Hrsg.), 1995: God at the Grass Roots: The Christian Right in the 1994 Elections, Lanham.

Rozell, Mark J./Wilcox, Clyde (Hrsg.), 1997: God at the Grass Roots: The Christian Right in the 1996 Elections, Lanham.

Rucht, Dieter (Hrsg.), 1991: Research on Social Movements. The State of the Art in Western Europe and the USA, Frankfurt a. M./Boulder.

Rucht, Dieter, 1993: Parteien, Verbände und Bewegungen als Systeme politischer Interessenvermittlung, in: Oskar Niedermayer/Richard Stöss (Hrsg.), Stand und Perspektiven der Parteienforschung in Deutschland, Opladen, 251-275. 
Rucht, Dieter, 1994: Modernisierung und neue soziale Bewegungen. Deutschland, Frankreich und USA im Vergleich, Frankfurt a. M./New York.

Rupp, Leila J./Taylor, Verta, 1987: Survival in the Doldrums. The American Women's Rights Movement, 1945 to the 1960s, New York/Oxford.

Ryan, Barbara, 1992: Feminism and the Women's Movement. Dynamics of Change in Social Movement Ideology and Activism, New York.

Sanbonmatsu, Kira, 1998: Easy Issues in the Party System: Gender, Race and Abortion. Paper presented at the Annual Meeting of the American Political Science Association, Boston.

Schmidt, Manfred G., 1995: Wörterbuch zur Politik, Stuttgart.

Schwartz, Felice N./Levine, Suzanne K., 1996: The Armchair Activist. Simple Yet Powerful Ways to Fight the Radical Right, New York.

Shaiko, Ronald G., 1999: Voices and Echoes for the Environment. Public Interest Representation in the 1990s and Beyond, New York.

Small, Melvin, 2002: Antiwarriors: The Vietnam War and the Battle for America's Hearts and Minds, Wilmington.

Snow, David A./Soule, Sarah Anne/Kriesi, Hanspeter (Hrsg.), 2004: The Blackwell Companion to Social Movements, Oxford.

Staggenborg, Suzanne, 1991: The Pro-Choice Movement. Organization and Activism in the Abortion Conflict, New York/Oxford.

Sundquist, James L., 1983: Dynamics of the Party System. Alignment and Realignment of Political Parties in the United States, Washington DC.

Vaid, Urvashi, 1995: Virtual Equality. The Mainstreaming of Gay and Lesbian Liberation, New York.

Wasser, Hartmut, 2007: Die Interessengruppen, in: Wolfgang Jäger/Christoph M. Haas/Wolfgang Welz (Hrsg.), Regierungssystem der USA, München/ Wien (3. Aufl.), 327-346.

Watson, Justin, 1997: The Christian Coalition. Dreams of Restoration, Demands for Recognition, New York.

Wattenberg, Martin P., 1991: The Rise of Candidate-Centered Politics. Presidential Elections of the 1980s, Cambridge.

Wattenberg, Martin P., 1996: The Decline of American Political Parties, 1952-1994, Cambridge.

Wieland, Leo, 2000: Bush lenkt die Republikaner in die politische Mitte. Vorsichtige Korrekturen am Programm, in: Frankfurter Allgemeine Zeitung, 31. Juli, S. 3.

Wilcox, Clyde, 2000: Onward Christian Soldiers? The Religious Right in American Politics, Boulder (2. Aufl.). 
Wolfe, Alan, 1983: Why is there no Green Party in the United States?, in: World Policy Journal 1, 159-180.

Zald, Mayer N./Useem, Bert, 1987: Movement and Countermovement Interaction: Mobilization, Tactics, and State Involvement, in: Mayer N. Zald/John D. McCarthy (Hrsg.), Social Movements in an Organizational Society. Collected Essays, New Brunswick, 247-272.

Korrespondenzanschrift:

Prof. Dr. Dr. Manfred Brocker

Lehrstuhl für Politische Theorie und Philosophie

Katholische Universität Eichstätt-Ingolstadt

Universitätsallee 1

85072 Eichstätt

E-Mail: manfred.brocker@ku-eichstaett.de

Web: http://www.ku-eichstaett.de/Fakultaeten/GGF/fachgebiete/Politikwissenschaften/Politikwissenschaft_II/Mitarbeiter/Brocker.de

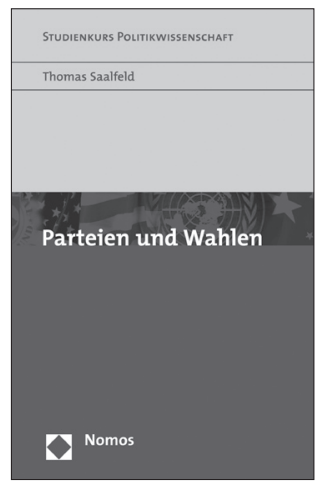

\section{Parteien und Wahlen}

Von Thomas Saalfeld

2007, 350 S., brosch., 21,90€, ISBN 978-3-8329-1064-8

(Studienkurs Politikwissenschaft)

Der Band analysiert die Rolle politischer Parteien in konsolidierten Demokratien. Dabei werden Parteien sowohl in ihrer Beziehung zu den Wählern als auch als Organisationen und in ihren Interaktionen auf der gouvernementalen Ebene behandelt. 